\title{
Stochastic electron motion driven by space plasma waves
}

\author{
G. V. Khazanov' ${ }^{1}$, A. A. Tel'nikhin ${ }^{2}$, and T. K. Kronberg ${ }^{2}$ \\ ${ }^{1}$ NASA Goddard Space FlightCenter, Greenbelt, MD, USA \\ ${ }^{2}$ Altai State University, Department of Physics and Technology, Barnaul, Russia \\ Correspondence to: G. V. Khazanov (george.v.khazanov@nasa.gov)
}

Received: 22 July 2013 - Revised: 19 November 2013 - Accepted: 21 November 2013 - Published: 10 January 2014

\begin{abstract}
Stochastic motion of relativistic electrons under conditions of the nonlinear resonance interaction of particles with space plasma waves is studied. Particular attention is given to the problem of the stability and variability of the Earth's radiation belts. It is found that the interaction between whistler-mode waves and radiation-belt electrons is likely to involve the same mechanism that is responsible for the dynamical balance between the accelerating process and relativistic electron precipitation events. We have also considered the efficiency of the mechanism of stochastic surfing acceleration of cosmic electrons at the supernova remnant shock front, and the accelerating process driven by a Langmuir wave packet in producing cosmic ray electrons. The dynamics of cosmic electrons is formulated in terms of a dissipative map involving the effect of synchrotron emission. We present analytical and numerical methods for studying Hamiltonian chaos and dissipative strange attractors, and for determining the heating extent and energy spectra.
\end{abstract}

\section{Introduction}

In this study, we treat the stochastic motion of charged particles resonantly interacting with nonlinear electromagnetic space plasma wave fields. Particular attention is given to the problem of nonlinear interaction between whistler mode waves and Earth's radiation belt electrons. Another problem of interest is the stochastic acceleration of cosmic wave electrons by space plasma waves at galactic shocks. We use a Hamiltonian formalism to treat these two problems. The Hamiltonian's equations of multiperiodical motion are assigned a measure-preserving map, the explicit form of which is defined by the closed set of nonlinear difference equations. The map is parameterized by some quantity, the control parameter, having a hard dependence on the structure of the wave packet and its power. Using topological arguments, we determine the value of the control parameter at which the system exhibits chaotic motion in the form of a strange attractor. In this case, every particle can explore the entire phase space energetically accessible to it; as a result, the upper bound of the strange attractor can be put on a one-to-one correspondence with the upper boundary of an energy spectrum whose value depends parametrically on the spectral power of the wave. The chaotic motion on the strange attractor is ergodic with mixing and as a consequence, the evolution of the distribution function and all means obeys the FokkerPlanck-Kolmogorov equation. As the wave power increases above some critical value, the phase space structure undergoes a change in topology called intermittency. The behavior is more complex, exhibiting random transitions between regular and stochastic motion. In this regime, diffusion in energy is realized through the drift of orbits in phase space. The generic Hamiltonian model is extended to include the effect of dissipation of energy associated with the synchrotron emission of relativistic electrons. It also proves possible to represent the dynamics of the system in the form of a dissipative map. For a dissipative system, the topology of attractors on which the motion appeared chaotic has the properties of fractional dimensionality. Another interesting effect in the Hamiltonian system occurs when an extrinsic noise is present. Numerical computations are presented to illustrate the methods and to give insight, and, also, to verify analytical results. We believe that acceleration mechanisms due to the nonlinear wave-particle interactions are capable of producing relativistic radiation belt electrons and galactic cosmic ray particles.

First, we will apply the model to the dynamics of Earth's radiation belts (RBs). It is suggested that wave-particle interactions, which are well correlated with the solar wind, may be responsible for the RB electrons. Energy diffusion due to resonance with chorus waves can be a viable mechanism for generating RB electrons, and the cyclotron resonances 
between electrons and chorus near the magnetic equator are thought to result in particles being scattered into the loss cone, forming bursts of precipitation. We show that significant heating rates and pitch angle diffusion occur for the RB electrons with energies from a few $\mathrm{keV}$ up to a few $\mathrm{MeV}$, and the calculated timescales and the total energy input to the atmosphere from relativistic electrons are in reasonable agreement with experimental data. This is the subject of Sect. 4. We deal with the problem of acceleration of cosmic rays in Sect. 5. High-energy particles observed in cosmic rays can be regarded as tails of the particle distribution in space plasmas. The energy relations show that collective plasma processes can play an important role in the evolution of the energy spectra of cosmic rays. The observations of synchrotron emission from shell supernova remnants (SNRs) have shown that most cosmic rays are produced by SNRs. We show that electrons can be accelerated up to high energies by the Langmuir and upper-hybrid electrostatic waves at the foot of the front of galactic shocks. We follow up by applying the model to stochastic surfing electron acceleration at galactic shocks to take into account the impact of synchrotron emission on the dynamics and to interpret the synchrotron emission spectrum.

\section{Dynamics of particles}

We will consider the dynamics of a charged particle resonantly interacting with space plasma wave fields. Hamiltonian formalism is applied to describe the dynamics in collisionless space plasmas. As known, the Hamiltonian formalism is based on the theory of smooth manifolds and differential geometry (Schutz, 1982; Arnold, 1988, 1989). In this approach any state of a dynamic system is given by a point in phase space. Let $2 \mathrm{n}-\mathrm{D}$ (dimensional) smooth manifold $M$ on which the Hamilton function $H(q, p)$ is defined,

$$
\left(q^{1}, q^{2}, \ldots, q^{n}, p_{1}, p_{2}, \ldots, p_{n}\right)=(q, p)
$$

are canonical coordinates, be the phase space of a system. Then dynamics is determined by the Hamiltonian vector field

$\boldsymbol{v}=\frac{\partial H}{\partial p_{\mu}} \frac{\partial}{\partial q^{\mu}}-\frac{\partial H}{\partial q^{\mu}} \frac{\partial}{\partial p_{\mu}}$,

where $\partial / \partial q$ and $\partial / \partial p$ are the basis vector fields, which act as linear differential operators on smooth functions. Equations (2) are equivalent to the phase flow given by the differential equations

$$
\begin{aligned}
\dot{q}^{\mu} & =\left[q^{\mu}, H\right]=\partial H / \partial p_{\mu}, \\
\dot{p}_{\mu} & =\left[p_{\mu}, H\right]=-\partial H / \partial q^{\mu}, \\
H(q, p: t) & =\sqrt{\left(p_{\mu}-A^{\mu}\right)\left(p^{\mu}-A_{\mu}\right)+m^{2}}+\varphi,
\end{aligned}
$$

where $\boldsymbol{p}$ is the canonical momentum, $\boldsymbol{A}$ the vector potential, $\varphi$ the scalar (electrostatic) potential of the wave field, $H$ is the Hamiltonian associated with the problem, and [ , ] stand for the Poisson brackets. We have employed here, and throughout this paper, the system of units in which the speed of light $c=1$ and the electron charge $|e|=1$.

For the field variables $\boldsymbol{A}$ and $\varphi$, which are explicit functions of coordinates and time, we have to specify the coordinate system. We have chosen a Cartesian spatial coordinate system whose $z$ axis is directed along the external magnetic field, and the plane perpendicular to the external magnetic field direction is spanned by the orthogonal coordinates $x$ and $y$. Now our manifold looks locally like a linear real space $R^{6}, q^{\mu}=(x, y, z) \in R^{3}, p_{\mu}=\left(p_{x}, p_{y}, p_{z}\right) \in R^{3}$, i.e., the local topology of $M$ is identical to that of the space. We make use of the Lorentz gauge, assuming

$\operatorname{div} \boldsymbol{A}_{\mathrm{t}}^{\mathrm{W}}=0, \quad \boldsymbol{A}=\left(-B y-A_{\mathrm{t}}, 0,0\right)$,

$\boldsymbol{B}_{\mathrm{t}}=\operatorname{rot} \boldsymbol{A}_{\mathrm{t}}, \quad \boldsymbol{E}_{\mathrm{t}}=-\partial \boldsymbol{A}_{t} / \partial t, \boldsymbol{E}_{l}=-\nabla \varphi$,

where the subscripts $t$ and 1 denote the transverse and longitudinal components of the electromagnetic wave field, and $-B y$ is the vector potential for constant external magnetic field $\boldsymbol{B}$ (Landau and Lifshitz, 1980). Then we write the wave field in the form of a transverse longitudinal wave with slowly varying amplitude

$\left(\begin{array}{c}A_{t} \\ \varphi\end{array}\right)=\left(\begin{array}{c}A(\varepsilon t, \varepsilon z) \\ U(\varepsilon t, \varepsilon z)\end{array}\right) \cos \left(k_{z} z+k_{t} y-\omega t\right)$.

The functions $A(\varepsilon t, \varepsilon z)$ and $U(\varepsilon t, \varepsilon z)$ describe the repetitive space-time structure of the envelope of the wave. Let the shape of the envelope be given by a smooth periodical function $f(\varepsilon t)$, such that

$f(t+T, z+L)=f(t, z)$,

$\varepsilon=\frac{\partial f}{\partial t} \cdot \frac{1}{\omega f} \sim(\omega T)^{-1}, \frac{\partial f}{\partial z} \cdot \frac{1}{k f} \sim(k L)^{-1}$,

where $\varepsilon$ is a small parameter, the ratio of the oscillation period $2 \pi / \omega$ (or $2 \pi / k)$ to the time (space) scale $(T, L)$, over which the envelope varies. This $\varepsilon$ also serves as an ordering parameter. Thus we assume the ambient magnetic field varies slowly over one wavelength $\varepsilon^{2}=\frac{1}{k_{z}} \partial \ln B / \partial z$, and the wave field is sufficiently small, $A / m, U / m=\varepsilon$.

We now take into account the axial symmetry of the nonperturbative problem, and introduce the new variables, an action $(I)$, and an angle $(\theta)$, by the canonical transformation,

$y=r \sin \theta, p_{y}=\left(m r \omega_{B}\right) \cos \theta ;$
$r=\sqrt{2 m \omega_{B} I} / m \omega_{B}, \omega_{B}=B / m$,

where $r$ is the gyroradius, and $\omega_{B}$ is the angular gyrofrequency, respectively (Sagdeev et al., 1988). The 
Hamiltonian (4) in this representation becomes

$$
\begin{aligned}
& H\left(z, p_{z} ; \theta, I ; t\right)=H_{0}\left(p_{z}, I\right) \\
& \quad+\left[\sqrt{2 m \omega_{B} I} H_{0}^{-1} A \sum_{n} J_{n}^{\prime}\left(k_{t} r\right)+U \sum_{n} J_{n}\left(k_{t} r\right)\right] \cos \psi, \\
& H_{0}\left(p_{z}, I\right)=\sqrt{m^{2}+p_{z}^{2}+2 m \omega_{B} I}, \\
& \psi=k_{z} z+n \theta-\omega t
\end{aligned}
$$

where $H_{0}\left(p_{z}, I\right)$ is the Hamiltonian of the non-perturbative system, and $\psi$ is the phase of the particle in the wave field. In deriving (12), the representation

$$
e^{i k_{t} r \cos \theta}=\sum_{n} J_{n}\left(k_{t} r\right) e^{i n \theta}
$$

has been employed, where $J_{n}(\cdot)$ are Bessel functions, $J_{n}^{\prime}$ are their derivatives with respect to the argument, and $n \in Z, Z$ is the set of all integers. We consider the case of resonance between the Doppler-shifted wave frequency and the gyromotion,

$\dot{\psi}=k_{z} \dot{z}+n \dot{\theta}-\omega=0$.

Resonant wave-particle interaction occurs whenever Eq. (16) holds, which is satisfied for a series of $n$ values for particles with different momentum and energy. Sufficiently close to a resonance, $\psi$ is slowly varying; therefore $p_{z}$ and $I$ are also slowly varying variables:

$\varepsilon=\dot{\psi} / \omega \psi, \dot{I} / \omega I, \dot{p}_{z} / \omega p_{z}$

This condition means that the adiabatic approach to the problem of resonant wave-particle interaction is applicable. Choosing a particular resonance, $n=s$, we can transform the Hamiltonian (12) as follows:

$$
\begin{aligned}
& H\left(z, p_{z} ; \theta, I ; t\right)=H_{0}\left(p_{z}, I\right) \\
& \quad+\left[\sqrt{2 m \omega_{B} I} H_{0}^{-1} \cdot A(\varepsilon t) J_{s}^{\prime}\left(k_{t} r\right)+U(\varepsilon t) J_{s}\left(k_{t} r\right)\right] \cos \psi, \\
& H_{0}\left(p_{z}, I\right)=\sqrt{m^{2}+p_{z}^{2}+2 m \omega_{B} I} \\
& \psi=k_{z} z+s \theta-\omega t
\end{aligned}
$$

where all of the perturbation terms average to zero except for $n=s$. Accordingly, we write the equations associated with the Hamiltonian (18) in the form

$$
\begin{aligned}
\dot{p}_{z} & =k_{z}\left(\sqrt{2 m \omega_{B} I} H_{0}^{-1} \cdot A J_{s}^{\prime}\left(k_{t} r\right)+U J_{s}\left(k_{t} r\right)\right) \sin \psi \\
\dot{I} & =s\left(\sqrt{2 m \omega_{B} I} H_{0}^{-1} \cdot A J_{s}^{\prime}\left(k_{t} r\right)+U J_{s}\left(k_{t} r\right)\right) \sin \psi \\
\dot{z} & =p_{z} / H_{0}, \dot{\theta}=\omega_{B} m / H_{0}
\end{aligned}
$$

We retain in these equations only the leading terms, where the small parameter $\varepsilon$ automatically keeps track of the ordering. Now our manifold looks locally like the real space
$R^{2} \times R \times S,\left(p_{z}, I\right) \in R^{2}, \dot{z} \in R, \theta \bmod 2 \pi \in S$. Because of the explicit symmetries of time translation and rotation of the phase, the phase space representation offers significant simplifications in treating the problem. Indeed, the Hamiltonian (18) is invariant under the transformations (8) and (10),

$H=H \exp (i \psi), H(t+T)=H(t)$,

consequently, the phase flow conserves the invariant of motion,

$s p_{z}-k_{z} I=$ const.

where const. is a constant independent of time, determined by the initial condition. This leads to the restriction of dynamics onto a reduced phase space, acceptable coordinates of which appear to be a canonically conjugated pair $(\psi, u)$, where $\psi$ is the phase variable, and $u$ is a new action variable. With these simplifications we can reduce this motion to quadratures only if the perturbation has a trivial form of monochromatic wave propagating along the direction of the ambient magnetic field. In the general case this nonautonomous nonlinear dynamical system is non-integrable, and the measure of its regular motions is equal to zero.

Consider the expanded phase space of the Hamiltonian system. Such a space has a natural structure of the bundle, the base of which is the discrete time $t_{n}=n T, n \in Z$, and the fiber is the $R \times S$ space (Schutz, 1982). In this case, it is sufficient to describe the motion on some time interval $\left(t_{0}, t_{0}+T\right)$, for example, $g^{1}\left(\psi_{0}, u_{0}\right)=\left(\psi_{1}, u_{1}\right)$, where $\left(\psi_{0}, u_{0}\right)$ is the initial state of the system, and $g^{1}$ is the map at one period (Arnold, 1989). In this way we have defined a map of the phase plane onto itself $g^{n}: R \times S \rightarrow R \times S$. The map is a diffeomorphism, which forms a one-parameter group $g^{n}=\left(g^{1}\right)^{n}$ of diffeomorphisms of the phase plane, and preserves the phase volume. This statement follows from Liouville's theorem. This bundle and the group $g^{n}$ acting on it are shown in Fig. 1.

For the Hamiltonian (18) and its invariant of motion (25), this group can be written in the form of a set of nonlinear difference equations,

$u_{n+1}=u_{n}+Q \sin \psi_{n}, \psi_{n+1}=\psi_{n}+F\left(u_{n+1}\right) \quad \bmod 2 \pi$,

where $Q$ is the control parameter, which defines the intensity of wave-particle interaction, and the function $F\left(u_{n+1}\right)$ describes the shift of phase acquired by a particle. The system (26) exhibits a rich variety of chaotic phenomena in various parameter regimes. This kind of dynamics is typically related to the appearance of a strange attractor (SA), which represents persistent chaotic motion over a global domain of phase space. In that sense, the SA is to be a paradigm for obtaining some information about the dynamics. This information is primarily geometrical and statistical, such as the fractal dimensions, spectrum of Liapunov exponents of the underlying SA and the invariant distribution on the SA. Any SA 


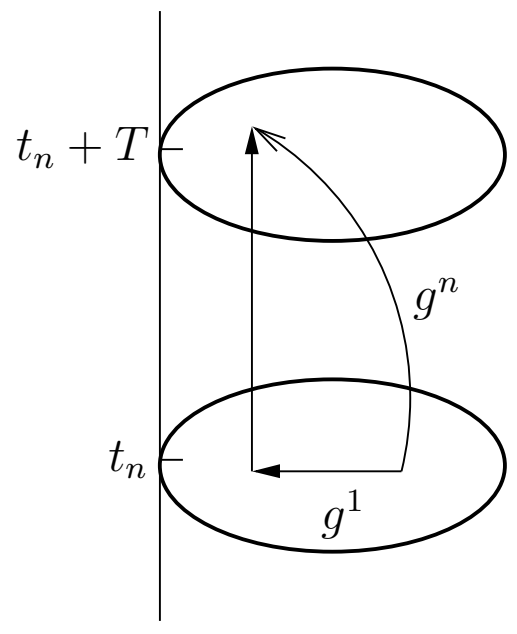

Fig. 1. Map at one period (after Arnold, 1988).

is known to be both ergodic and mixing (Arnold and Avez, 1968), and for many dynamical systems of interest, the phase variable randomizes much more rapidly than the action variable $u$, allowing kinetic description in terms of a standard Fokker-Planck-Kolmogorov (FPK) equation for the distribution $w(u)$ alone. For area-preserving maps, the distribution on any SA is trivially a constant. A common procedure for finding $w(u)$ consists of iterating an initial state, or of solving an FPK equation. Applying the invariant distribution, we may replace time averages by phase-space averages in calculating the steady state value of a physical observable. If $G(u)$ is an observable function in phase space, then the phase average

$\langle G\rangle=\int d u w(u) G(u)$

is independent of $u_{0}$ (for almost all initial values $u_{0}$ in the basin of a given SA). We are obliged to say strange attractor; now we should wish to prove that these kinds of objects are common in the problem of interest, so we will try in the subsequent sections to develop all the proofs as explicitly as possible.

\section{Dynamics of wave fields}

We are going to inspect the problem of dynamics of particles in space plasma wave fields. The first question of course is how to describe these wave fields. A common technique in studying the behavior of wave fields is to Fourier analyze transform into mode amplitudes, to obtain an equation of motion for each mode. If only the $N$ most important modes are kept, this motion is given by a set of ordinary differential equations describing the evolution in time of the mode amplitude components. This procedure of Fourier analysis followed by truncation is called the Galerkin approximation. Any stationary nonlinear wave is known to be a strongly exited state of a nonlinear medium, which emerges due to the competition between dispersion and nonlinearity. Such a wave represents in itself the bound state of a large number of harmonics. It is worthwhile emphasizing that these are not true modes of the nonlinear system, and thus interchange of energy among these quasimodes will occur even in the absence of external noise. There exists a number $N$ that actually truncates the wave spectrum exponentially, and this $N$ is, in fact, the constant of coupling for stationary nonlinear waves (Sagdeev et al., 1988).

Another approach to describing wave fields consists of the proper choice of basis wave models in the standard form of nonlinear wave equations. For instance, the applicability of the Korteweg-de Vriez equation for describing nonlinear space plasma phenomena is well established. In that case, the nonlinear dispersion relation, which includes the main part of information about wave dynamics, is what we chiefly need (Bernstein et al., 1957; Lutomirski and Sudan, 1966). A general approach for deriving nonlinear dispersion relations has been developed in different techniques, by Whitham (1974), Karpman (1975), and Kaup and Newell (1978). They have shown that any nonlinear dispersion relation conserves its own form even if the wave propagates in a weakly inhomogeneous and weakly nonstationary medium. This important conclusion follows from the strong stability of a nonlinear wave packet. The basic model for modulated waves in a dispersive medium appears to be the familiar nonlinear Schrödinger (NLS) equation,

$$
\begin{aligned}
i\left(\partial \Phi / \partial t+v_{\mathrm{g}} \partial \Phi / \partial z\right)+\mu \partial^{2} \Phi / \partial z^{2}+ & \left(v_{\mathrm{g}} / 2 k_{0}\right) \triangle_{\perp} \Phi \\
& -g\left|\Phi^{2}\right| \Phi=0,
\end{aligned}
$$

for the complex wave amplitude $\Phi$ (Karpman, 1975). Here the group velocity $v_{\mathrm{g}}$ of the wave packet, and the parameters of dispersion $\mu$ and nonlinearity $g$ are determined entirely by a nonlinear dispersion relation $\omega=\omega\left(k^{2},|\Phi|^{2}\right)$ in the usual way,

$v_{\mathrm{g}}=(\partial \omega / \partial k)_{k_{0}}, \mu=\frac{1}{2}\left(\partial v_{\mathrm{g}} / \partial k\right)_{k_{0}}, g=\left(\partial \omega / \partial|\Phi|^{2}\right)_{k_{0}}$.

The subscript denotes that we have to put these values at $k=k_{0}$, where $k_{0}$ is the characteristic wavenumber. The basic nonlinear mechanism in this case is the ponderomotive force that arises through the coupling of the high- and the low-frequency oscillations, and numerous possibilities of the parametric decay and the process of modulation instabilities (Sagdeev et al., 1988). Equation (28), involving the effects of self-focusing and self-compressing, describes specifically the dynamics of Langmuir, upper-hybrid mode and whistler mode waves, i.e., the very waves that usually are detected (or inferred) in space plasmas and are likely to play a major role in stochastic heating of particles. For application purposes we discuss the relevance of the representations to the whistler wave events in the Earth's magnetosphere. The spatio-temporal structure chorus will need to describe the 
interaction and compare obtained results with experimental data. We will rely on the data represented by the CLUSTER mission (Santolik et al., 2003), which provided temporally high-resolution measurements of the wave events. In this work, chorus emissions have been measured at a radial distance of 4.4 Earth radii within a $2000 \mathrm{~km}$ region located close to the Equator. The wave vector direction in this region is nearly parallel to the field lines, and the waveforms of the packet show a fine structure consisting of subpackets with amplitudes above $300 \mathrm{pT}$. In each wave packet, the frequency changes with a typical rate of a few $\mathrm{kHzs}^{-1}$, and the spectrum is organized into two bands between 2 and $6 \mathrm{kHz}$. The spectral power $P$ of the wave has its peak value $P \sim 10^{-4} \mathrm{nT}^{2} \mathrm{~Hz}^{-1}$ at the frequency $3.8 \mathrm{kHz}$, close to the half-gyrofrequency $\omega_{B} / 2$. The chorus emission, being a coherent whistler mode, has a slowly varying amplitude (envelope), with the time period $T=200 \mathrm{~ms}$ determining the repetitive temporal structure of the chorus.

In order to interpret these results, we will use solutions of a nonlinear Schrödinger (NLS) equation, the applicability of which to whistler mode waves is well grounded (Karpman, 1975). In the wave frame, Eq. (28), for quasi-plane waves, takes the form

$i \Phi_{t}+\mu \Phi_{z z}-g|\Phi|^{2} \Phi=0$,

with $\Phi=b \exp i S$, where $b=B^{\mathrm{w}} / B$ is the wave amplitude, normalized to the external magnetic field $\boldsymbol{B}$, and $S$ is the eikonal of the wave. It is known (Karpman, 1975) that an NLS equation has several different classes of solutions. Of these, the most stable solutions are the so-called twoparameter envelope solitons

$$
\begin{aligned}
b(z-v t) & =a \operatorname{sch}(Q z-F t), \\
a^{2} & =F^{2} / G, \\
F & =Q v, G=\omega_{0} g,
\end{aligned}
$$

which are parameterized by the soliton velocity $v$ and by the soliton spacescale $L=1 / Q$. We will assume the value of $v$ to be close to $v_{\mathrm{g}}$. These solutions describe a nonlinear wave with a dispersion law

$\omega(k)=\omega_{0}(k)+g a^{2} / 2$,

where $\omega_{0}(k)$ is the linear dispersion relation for whistlers

$\omega_{0}(k)=\omega_{B} k^{2} c^{2} /\left(\omega_{p}^{2}+k^{2} c^{2}\right)$,

and $g a^{2} / 2$ is the nonlinear frequency shift. The NLS equation is completely integrable and possesses an entire set of integrals of motion. One of them,

$P=\int b^{2} \mathrm{~d} t$

is physically significant in virtue of the relation

$P=(1 / 2 \pi) \int b^{2}(\omega) \mathrm{d} \omega$, which determines the spectral power $b^{2}(\omega) /(2 \pi)$. Substituting Eq. (31) in the expression (36), we obtain

$P=2 a^{2} / F=2 F / G$

and, a Fourier transform of Eq. (31) gives

$b^{2}(\omega) /(2 \pi)=(\pi P / 4 F) \operatorname{sch}^{2}(\pi \omega / 2 F)$.

The spectral representation means that the nonlinear wave can be interpreted as a nonlinear wave packet whose amplitude and width $\Delta \omega=4 F / \pi$, unlike a usual wave packet, are bound with the coupling. It is known that any spatially localized perturbations to an NLS equation will convert eventually into a set of solitons. This result has been proved by the technique of inverse scattering transform (IST) (Newell, 1985). According to the IST, solitons of the NLS equations play the role of harmonic waves in linear systems, and the representation of solutions in the form of a set of solitons corresponds to a Fourier transform in linear equations. So the $N$-soliton solution of the NLS equation is

$$
\begin{aligned}
b(z, t) & =\sum_{i=1}^{N_{\mathrm{s}}} a_{i} \operatorname{sch}\left(Q_{i} z-F_{i} t\right), \\
a_{i}^{2} & =F_{i}^{2} / G,
\end{aligned}
$$

where $N_{\mathrm{s}}$ determines the total number of solitons. The IST involves quasi-classical quantization, and solutions (40) belong to the discrete spectrum of values of $a$ and $F$. The solitons of the NLS equation conserve physically significant properties, so the total spectral power is the sum of spectral powers of the single solitons,

$P=\sum_{i} P_{i}=2 \Delta F / G, \Delta F=\sum F_{i}$,

where $\Delta F$ is the half-width of a power spectrum. To interpret the spatio-temporal wave structure measured by Santolik et al. (2003), we apply the results of theoretical analysis to these data. We have approximated the temporal structure of chorus presented by Santolik et al. (2003) in Fig. 2 by the $N$-soliton solution of the type $E^{\mathrm{w}}=\sum_{i} E_{i} \operatorname{sch}\left(t / T_{i}\right)$ with $T_{i}=(14.37,1.18,0.89,1.03,0.84) \mathrm{ms}$, where $T_{i}$ are the timescales of each subpacket. The fitted values of the waveforms were obtained from the CLUSTER data as shown in Fig. 2. Figure 2 has been added to identify the wave's fine structure. There is an apparent correspondence between the waveforms described by the envelope soliton solutions and the experimental data. This gives us confidence to assume that those signals are at least close to the waveforms having the $N$-soliton envelope. As a result, we will assume the representation (40) is adequate for the waveform of a single wave packet, where we have to accomplish the substitution $t \rightarrow t-n T$, to describe the quasi-periodical structure of the chorus emission. The physical reason for the chirp effect is supposed to be the nonlinear dispersion of phase velocity. 
(a)

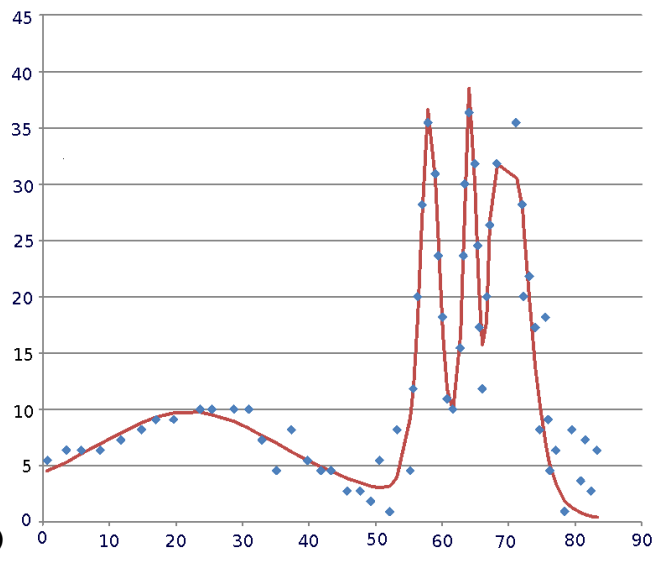

(b)

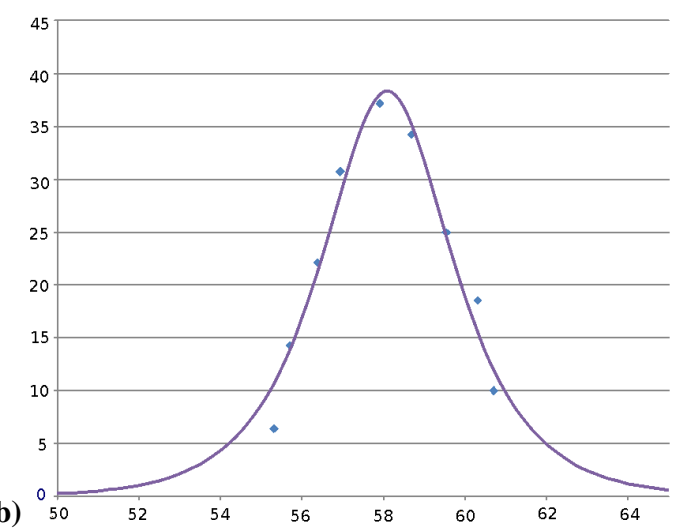

Fig. 2. (a) Single wave packet with the envelope approximated by $\mathrm{N}$-soliton solution. (b) Smooth curve given by sch $(t / 1.18 \mathrm{~ms})$ corresponds to the 2 th subpacket.

Indeed, it can be shown, any gradients of physical fields will lead to the change of wave vector: $\partial k / \partial t=-\partial \omega / \partial z$. Considering this expression and the nonlinear wave dispersion (34), we can obtain the equation of transfer of wave information

$\left(\frac{\partial}{\partial t}+v_{\mathrm{g}} \frac{\partial}{\partial z}\right) \omega=0$

As a consequence, we conclude that the nonlinear dispersion relation is always the same along the group velocity vector, $\left(\frac{\partial}{\partial t}, v_{\mathrm{g}} \frac{\partial}{\partial z}\right)$, even if the frequency (and wavenumber) changes slowly over the time period of the wave, $2 \pi / \omega$, such that

$\dot{\omega} 2 \pi / \omega \ll 1 \dot{\omega}=\partial \omega / \partial t$.

Then taking into account the nonlinear wave dispersion (34), one computes the wave drift rate, $\dot{\omega}=(g / 2) \partial a^{2} / \partial t$. Applying to this the soliton-like solution for an envelope, the detailed evolution of the wave frequency (exponential growth of frequency on the initial stage, nonlinear dependence on the wave amplitude, etc.) can be studied. The mean and maximal rates are evaluated as $\dot{\omega} \sim g a^{2} / T \sim F^{2} /(\omega T)$, and $\dot{\omega}_{\max } \sim$ $g a^{2} F / 3 \sim F^{3} /(3 \omega)$. One calculates $F=\sqrt{g a^{2} \omega}$, with the help of Eq. (41), then puts together the empiric data (Santolik et al., 2003); we evaluate $g a^{2} / 2 \sim 200 \mathrm{~Hz}, F \sim 1,2 \mathrm{kHz}$, and therefore, $\langle\dot{\omega}\rangle \sim 2 \mathrm{kHzs}^{-1}$ and $\dot{\omega}_{\max } \sim 100 \mathrm{kHzs}^{-1}$. Thus we explicitly show that the condition (44) holds. Note that longitudinal inhomogeneities of the ambient magnetic field $\boldsymbol{B}(z)$ and particle density $N_{\mathrm{e}}(z)$ do not influence the effect because the fundamental mode $\omega_{0}=\omega_{B} k^{2} c^{2} / \omega_{p}^{2}$ is proportional to $B / N_{\mathrm{e}}$, and this ratio is in fact a constant along a force line near the equatorial plane. The rising tone of chorus is observed in the region of generation and formation of waveforms due to self-modulation and, due to the propagation of waves away from this region when the modulation instability is stabilized, this effect may vanish, as was noted by Cully et al. (2008) and Wilson et al. (2011). The spectral gap is likely due to just the same competition between nonlinearity and dispersion under conditions of the exact cyclotron and Landau resonance at $\omega=\omega_{B} / 2, v_{\mathrm{r}}=v_{\mathrm{p}}$. Presumably the chirp-effect and spectral gap are rather the manifestation of intrinsic properties of the typical nonlinear whistler mode wave rather than a new type of emission.

The effect of diffraction on wave propagation is described by the third term of the NLS equation (28). Let AD be the angle of diffraction, $\mathrm{AD}=2 / k d$, where $d$ is the perpendicular size of the region of wave generation, and $k$ is the wave number. In accordance with (Santolik et al., 2003), AD 0.1 and these waves propagate from their source nearly parallel to the field line. Putting $\mathrm{AD}=0.1, k=0.25 \mathrm{~km}^{-1}$, we will have an estimate of $d, d \sim 100 \mathrm{~km}$, and the lengthscale of the wave field, $l_{d}=k d^{2} / 2, l_{d} \approx 2000 \mathrm{~km}$, which is comparable with the spacescale of the envelope. At $z=0$ the wave beam has a plane phase front and a Gaussian distribution of amplitude $b(r)=b(0) \exp \left(-r^{2} / d^{2}\right)$. The solution of the equation shows that the width of the packet grows as $d^{2}(z)=d^{2}\left(1+\left(z / l_{d}\right)^{2}\right)$, and the radius of curvature grows as $R=z+l_{d}^{2} / z$. As $z / l_{d} \gg 1$; the originally plane wave becomes spherically divergent, its amplitude decreases as $k d^{2} b(0) / 2 z$, and its width grows as $d(z) \approx 2 z / k d$. For example, if the wave was generated at a large $L$ and traveled a great distance to reach Polar, this effect will lead to a lower intensity at Polar (Tsurutani et al., 2011).

As noted above, the impact of the inhomogeneity of the ambient magnetic field and density fields on the wave propagation is negligible because of a constancy of the ratio $B / N_{\mathrm{e}}$. The solutions of an NLS equation, in particular with a parabolic profile of perturbations, are well known (Newell, 1985). According to Newell (1985), the wave power spectrum and shape remain unchanged if the envelope's size (intrinsic spacescale) is less than the spacescale of an external field. Typically, this condition is easy satisfied. Another source of perturbations can be the spacecraft itself. As long as the size of the spacecraft is much less than the wavelength, the effect of imperfection of the wave can be described as the scattering of a soliton-like packet on this defect. This effect is local, therefore it immediately appears in the wave 
measurements (Hastings and Garrett, 1996). It is possible that it helps explain the modification of waveforms in wave events (Santolik et al., 2003).

Another powerful tool, especially when the explicit shape of a nonlinear wave is unknown, is spectral representation in the so-called form of time- and space-like wave packets (Sagdeev et al., 1988). Let $f(t)$ be a periodic function, $f(t+$ $T)=f(t)$, given by the convergent Fourier series

$f(t)=\sum_{n \in Z} f_{n} e^{-i \omega_{n} t}, \omega_{n}=2 \pi n / T$,

whose coefficients $f_{n}$ satisfy the Parceval identity,

$\sum_{n}\left|f_{n}\right|^{2}=\left\langle f^{2}(t)\right\rangle,\left\langle f^{2}\right\rangle=\frac{1}{2 T} \int_{-T}^{T} f^{2}(t) \mathrm{d} t$.

Let $f(t)$ correspond to a certain realization of a physical process. Then the measurable quantity is $\left\langle f^{2}\right\rangle$, and/or its spectral density $\sum_{n}\left|f_{n}\right|^{2}$. One assumes this function characterizes the intensity of a nonlinear wave packet. As mentioned above, each packet is a bound state of $N$ quasimodes, and $N$ takes part a constant of coupling. The implication of this is that as interchange of energy among these quasimodes occurs, the constant wave power, proportional to $\left\langle f^{2}\right\rangle$, will share evenly among the quasimodes. In virtue of Eq. (46), we obtain $\sum_{n}\left|f_{n}\right|^{2} \sim N f_{\mathrm{r}}^{2} \sim$ const., and consequently, $f_{\mathrm{r}}=$ $f_{0} / \sqrt{N}$, where the value of $f_{0}$ would have to be extracted from a measured physical quantity, for instance, the spectral density. A more rigorous result, obtained in the theory of distributions, states the following (Richtmyer, 1978). Let $f(t)$ be a generalized function such that

$f(\tau+1)=f(\tau), e^{i 2 \pi \tau} f(\tau)=f(\tau), \tau=t / T$,

then, this function is found to be

$f(\tau)=$ const. $\sum_{n \in Z} \delta(\tau-n)$, const. $=f_{\mathrm{r}}$.

The wide wavepacket approach follows from these statements and the physical meaning of the theory of nonlinear waves (Sagdeev et al., 1988). Taking the structure of a wide wave packet to be of the form

$A(\varepsilon t, \varepsilon z)=\sum_{n} A_{n} \sin (n \delta k z-n \delta \omega t)$,

where

$\delta k=2 \pi / L, \delta \omega=2 \pi / T$,

$L, T$ are the length and time scales of the problem. Then we suppose that all spectral characteristics $A_{n}$ are equalamplitude, and write the envelope of wave packet in the form

$$
\begin{gathered}
A(\varepsilon t, \varepsilon z)=A_{\mathrm{r}} \sum_{n} \sin (n \delta k z-n \delta \omega t), \\
A_{\mathrm{r}}=A_{0} / \sqrt{N}, N=\Delta v T,
\end{gathered}
$$

where $A_{0}$ is the characteristic amplitude of the wave, and $\Delta v$ is the width of the frequency spectrum. In the limit $\delta k \rightarrow 0$, expression (51) can be transformed into

$A(\varepsilon t, \varepsilon z)=A_{\mathrm{r}} \sum_{n \in Z} \delta(t / T-n)$.

There is a time-like representation (TLR) of a wide wave packet. In deriving it we have used the relation

$\sum e^{\mathrm{inx}}=2 \pi \sum \delta(x-2 \pi n)$,

where $\delta(\cdot)$ is the Dirac delta function. Carrying out the same procedure with respect to Eq. (51), in another limit $\delta \omega \rightarrow$ 0 , the expression takes the form of a space-like (SL) wave packet

$A(\varepsilon t, \varepsilon z)=A_{\mathrm{r}} \sum_{n \in Z} \delta(z / L-n)$.

\section{Whistler-electron interaction in the Earth's radiation belts}

Wave-particle interaction with chorus is believed to be the main mechanism for the acceleration of $10-100 \mathrm{keV}$ radiation belt (RB) electrons to relativistic energies, a major topic in space weather physics today. In the area of investigations, progress is related to highly resolved wave measurements of intensive chorus (Santolik et al., 2003; Tsurutani et al., 2011) and oblique large amplitude whistlers (Cattell et al., 2008) as well as observations of relativistic electron microburst precipitation events (Blake et al., 1997). These results show that the usual quasilinear models of electron energizations and scattering via small amplitude incoherent waves (Summers et al., 2007) may be inadequate for understanding radiation belt dynamics. There is a need to develop a new theory for wave-particle interaction between RB electrons and coherent whistler mode waves. Some aspects of the problem have been treated by Faith et al. (1997), Roth et al. (1999), Wykes et al. (2001), and Albert (2002), who showed that the nonlinear coupling between a monochromatic whistler wave and the bouncing motion of RB electrons may lead to a random walk in energy and pitch angle. In this case, as shown by Faith et al. (1997), an electron gains energy only chaotically, when the wave field exceeds a threshold value. These results hinted that this mechanism may be promising in the many applications where the efficiency of particle heating is of prime importance. In particular, these resonant random processes may contribute to the 10 - to 100 -fold increase in the relativistic electron fluxes in the RBs, as well as constitute an interesting relation between wave activity and enhancements in fluxes during geomagnetic storms and other active periods (Roth et al., 1999). Finally, the concept of a strange attractor (SA) was introduced by Khazanov et al. (2007) and Khazanov et al. (2008a) in order to describe chaotic motion of RB electrons under the conditions of nonlinear resonant interaction with chorus. Strange attractors, which embody the idea 
of deterministic chaos, the statistical aspects of SAs have come under explicit study. The method has proved fruitful in describing the dynamics of electrons in the Earth and Jovian radiation belts.

\subsection{Chaotic motion of the RB electrons driven by chorus}

We are concerned with the dynamics of nonrelativistic electrons in the whistler mode chorus, which accelerates particles to relativistic energies through the resonance

$-k_{z}\left|v_{z}\right|+\omega_{B}-\omega=0$

Taking into account the relation $\omega_{B} I=m v_{t}^{2} / 2$, we write the invariant of Hamiltonian flow (25) as

$v_{t}^{2}=2\left(\omega_{B} / \omega\right) v_{\mathrm{ph}}\left(v_{\mathrm{r}}-\left|v_{z}\right|\right)$,

where $v_{\mathrm{r}}=v_{\mathrm{ph}}\left(\omega_{B}-\omega\right) / \omega$ is the resonance speed of an electron, and $v_{t}$ is the perpendicular (to $\boldsymbol{B}$ ) velocity of an electron. Then the equations of motion (21)-(23) can be represented in the form

$\dot{v}_{t}=\left(\omega_{B}^{2} / 2 \omega\right) v_{\mathrm{ph}}(b / \sqrt{N}) T \sin \psi \sum_{n \in Z} \delta(t-n T)$,

$\dot{\psi}=\omega\left(v_{t}, \omega\right)=\omega_{B}-\omega-k_{z} v_{\mathrm{r}}+\left(k^{2} / 2 \omega_{B}\right) v_{t}^{2}$.

We have also employed the invariant of motion, the value of the Bessel function $J_{1}^{\prime}(0)=1 / 2$ and the relationships

$A / m=\left(\omega_{B} / \omega\right) v_{\mathrm{ph}} b, \quad b=B_{t} / B$

After integrating these equations, the problem is formulated in terms of a $G^{n}$ map, given by a closed pair of nonlinear difference equations,

$G^{n}: \begin{aligned} & u_{n+1}=u_{n}+Q \sin \psi_{n}, \\ & \psi_{n+1}=\psi_{n}+\frac{1}{4} \omega T u_{n+1}^{2} \operatorname{sign} u \bmod 2 \pi,\end{aligned}$

where the new variable $u$ and the control parameter $Q$ have been introduced by the relations

$u=v_{t} / v_{\mathrm{r}}, Q=\left(\omega_{B}^{2} / 2 \omega\right) \sqrt{T P}=\omega_{B} T\left(\frac{\omega_{B}}{2 \omega}\right) \frac{b}{\sqrt{N}}$,

and $P$ is the wave power normalized to $B^{2}$. The map (59) acts as the group $G^{n}=\left(G^{1}\right)^{n}$ of diffeomorphisms of phase space, hence the pair $\left(M, G^{n}\right)$, together with the invariant of motion is equivalent to the periodic flow (21)-(23). Khazanov et al. (2008a) and Khazanov et al. (2011) have shown that the representation (52) is tantamount to that given by a solution of nonlinear wave Eq. (40). The physical explanation of this somewhat surprising result is quite clear. Any periodic, nonlinear wave can be described as wave packet consisting of some number $N$ of quasimodes with the frequency spacing $\delta \omega \sim 1 / T$, where $T$ is the time period of the envelope. In the situation to which we refer, the half-width
$\Delta v$ is about $1 \mathrm{kHz}, T$ is no less than $100 \mathrm{~ms}$, and therefore $N \sim \Delta v T\left(\sim 10^{2}\right)$. On the other hand, $\Delta v \sim \omega$, which indicates that $1 / N \sim \varepsilon$, the small parameter given by Eq. (9). Thus we determine that the representation (52) is reasonable, so the results obtained in these approaches either coincide or differ only slightly.

To understand the physical nature of these results, we examine the dynamics of a particle resonantly interacting with some quasimode of the wave packet. According to Eq. (57), the motion is governed by the pair of closed equations

$\dot{v}_{t}=\left(\omega_{B}^{2} / 2 \omega\right) v_{\mathrm{ph}}(b / \sqrt{N}) \sin \psi$,

$\dot{\psi}=\left(k^{2} / 2 \omega_{B}\right) v_{t}^{2}$.

Setting $v_{t}=v_{\mathrm{c}}+\Delta v$, where $v_{\mathrm{c}}$ is the value of $v_{t}$ in the resonance state, and linearizing this equation, we derive the equations

$\ddot{\psi}+\omega_{b}^{2} \sin \psi=0$,

$\omega_{b}^{2}=\omega \omega_{B} b / \sqrt{N}\left(v_{\mathrm{c}} / 2 v_{\mathrm{p}}\right)$,

which describes the small (bounce) oscillations of the particle with the frequency $\omega_{\mathrm{b}}$. Now we apply the overlapping criterion (Chirikov, 1979), $\omega_{\mathrm{b}} \geq \delta \omega\left(\sim T^{-1}\right)$, to obtain the condition of the onset of stochasticity,

$\left(v_{\mathrm{c}} / v_{\mathrm{p}}\right) \geq\left(2 \omega / \omega_{\mathrm{b}}\right) \sqrt{N} / b \omega^{2} T^{2}$.

Note that the criterion can be rewritten in the form $T_{\mathrm{b}} \leq T$, where $T_{\mathrm{b}}\left(\sim \omega_{\mathrm{b}}^{-1}\right)$ is the bounce period of the particle in the wave field. In amplitude-time terms, this relation determines the condition for parametric resonance between the slow variations in the wave field and the proper oscillations of a particle in the field. Thus the basic resonances (55) and their interaction through parametric resonance play a crucial role in the appearance of stochastic motion.

Our interest now is to inspect the motion of relativistic electrons, driven by chorus. By integrating Eqs. (21)-(23) along with invariant of motion (25), we derive in the usual way the $g^{n}$ map,

$g^{n}: \begin{aligned} & u_{n+1}=u_{n}+Q \sin \psi_{n}, \\ & \psi_{n+1}=\psi_{n}+(3 / 2) K u_{n+1}^{-2 / 3} \operatorname{sign} u \bmod 2 \pi,\end{aligned}$

written in the notations

$p_{z} / m=p, u=p^{3 / 2}$, and $K=v_{\mathrm{g}} \omega_{B} T$.

Here the control parameter $Q$ is given by

$Q=3\left(\omega_{B} v_{\mathrm{ph}} / 2 \omega\right)^{1 / 2} v_{\mathrm{g}} \omega_{B}(P T)^{1 / 2}$,

$P=b^{2} / \Delta v, b=B_{t} / B$.

Solutions of that map have been discussed in detail by the authors (Khazanov et al., 2008a, 2011). Let us consider a 
pair $\left(M, g^{n}\right)$, where $M$ is a smooth manifold, and $g^{n}$ is a diffeomorphism. Denote by

$J=\frac{\partial\left(u_{n+1}, \psi_{n+1}\right)}{\partial\left(u_{n}, \psi_{n}\right)}$

the Jacobi matrix of the map, the eigenvalues of which are given by the relations

$$
\begin{aligned}
\operatorname{det} J & =\lambda_{1} \cdot \lambda_{2}=1, \\
\operatorname{tr} J & =\lambda_{1}+\lambda_{2}=2+K Q u^{-5 / 3},
\end{aligned}
$$

where det $J$ and $\operatorname{tr} J$ denote the determinant and the trace of the matrix, respectively. The Jacobian of (65) is equal to one, and therefore $g^{n}$ has the structure of a differentiable areapreserving map and $(\psi, u)$ is the canonical pair of variables. It is known (Arnold and Avez, 1968) that the the geometrical structure of $M$ and dynamics of $g^{n}$ on $M$ are intimately closed, and that the condition

$$
|\operatorname{tr} J|=3
$$

corresponds to a topological modification at which the dynamics of the system becomes chaotic. In this situation, a single phase trajectory investigates all accessible phase space, which is called the strange attractor (SA). The strange attractor is the invariant set $g^{n} \mathrm{SA}=\mathrm{SA}, n \rightarrow \infty$ tightly embedded in phase space. The motion on any SA is random over a wide range of $Q$ due to the global stability of the SA, on which all means (observables) are stable indifferent of any (reasonable) initial conditions. Then we apply the condition of Eq. (67) to the relation (66) to obtain the upper bound of $\{u\}$

$u_{\mathrm{b}}=(K Q)^{3 / 5}$.

Shown in Fig. 3 is the strange attractor of the pair $\left(M, g^{n}\right)$. Figure 3 shows that the stochastic region extends to the values of $u$ predicted by Eq. (68).

Finally, using the relations (63) and (68) along with invariant of motion (25), we find the $Q$ dependence of the upper boundary of energy spectrum $E_{\mathrm{b}}$,

$$
E_{\mathrm{b}}=m(K Q)^{2 / 5} \text {. }
$$

At typical values of parameters, $\omega_{B} / \omega=2, \omega_{B} / 2 \pi=8 \times$ $10^{3} \mathrm{~Hz}, \quad P=10^{-4} \mathrm{nT}^{2} \mathrm{~Hz}^{-1}, \quad B=300 \mathrm{nT}, T=2 \times 10^{-1} \mathrm{~s}$ (Santolik et al., 2003), the expression (69) yields $E_{\mathrm{b}} \simeq$ $8 \mathrm{MeV}$. In this way as above, we can say the same about the pair $\left(M, G^{n}\right)$, just changing the order of words a little. Indeed, in this case, the condition (67) proves to determine the lower bound of $\{u\}$,

$u_{\mathrm{c}}=4 / \omega_{B}^{2} T \sqrt{P T}$,

which is in good agreement with the numerical solutions and results of qualitative analysis. With the values of the parameters as given above, the expression (70) yields $u_{\mathrm{c}} \approx 1 \times 10^{-3}$.
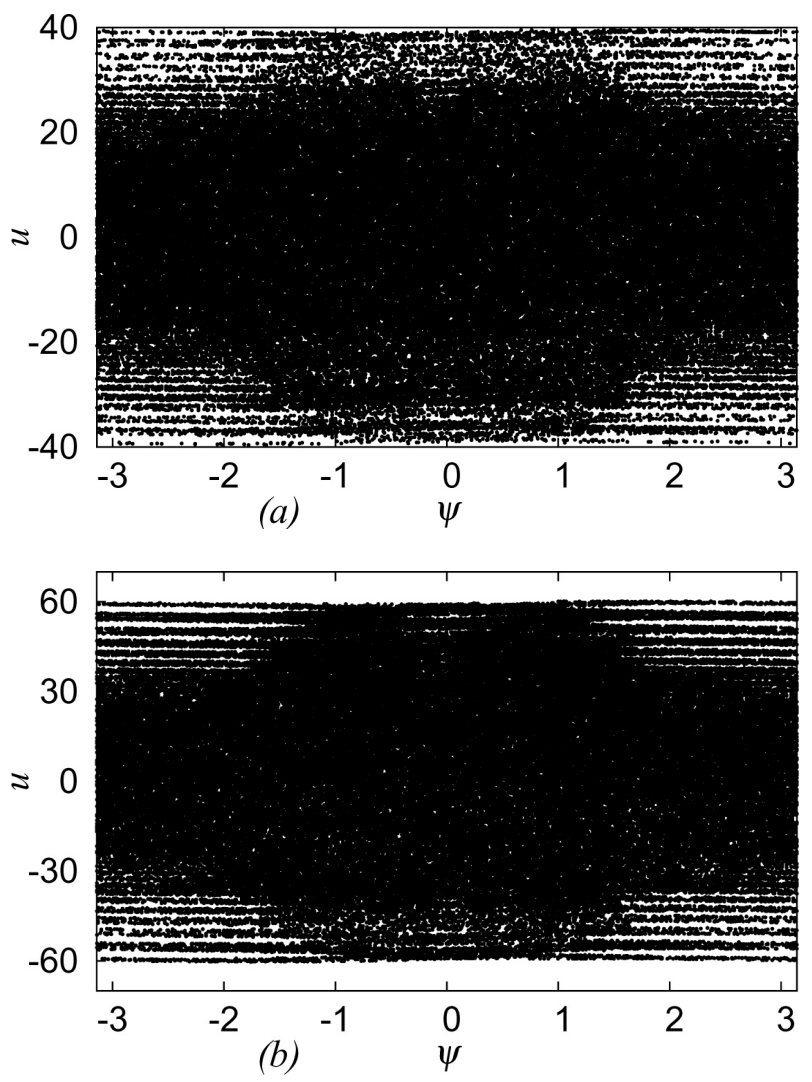

Fig. 3. Phase space for the map $g^{n}$ after $2 \times 10^{6}$ iterations with (a) $Q=0.5$ and (b) $Q=0.98$.

The phase space of the map $G^{n}$ is shown in Fig. 4. The overall picture of the phase space is quite different for $u<u_{\mathrm{c}}$ and $u>u_{\mathrm{c}}$. In the first case, the motion is regular. Figure 4 indicates the existence of a threshold for the initial particle velocity above which the trajectory becomes chaotic. We assume that nonlinear electron acceleration by a wave packet of whistler mode waves is always a stochastic process.

Now using the invariant of motion (56) and resonance condition (55), we have the relationship

$e=\left(1+u^{2} / 4\right)^{2}$

where $e=E / E_{\mathrm{r}}$ is the particle energy normalized to that at exact resonance $\left(E_{\mathrm{r}} \simeq 25 \mathrm{keV}\right)$. Putting in the expression (71) $u=u_{\mathrm{c}}$ and $u_{0}=\sqrt{2 \omega_{B} / \omega}$, we determine the range of heating $e \in\left(e_{\mathrm{c}}, e_{0}\right)$, where

$e_{\mathrm{c}} \simeq 1+u_{\mathrm{c}}^{2} / 2, e_{0}=\left(1+\omega_{B} / 2 \omega\right)^{2}$,

and evaluate (72) as $E_{\mathrm{c}} \sim 25 \mathrm{keV}, E_{0} \sim 100 \mathrm{keV}$. Lastly, the expression (72) is taken as a criterion that stochastic motion occurs, making available reliable information about the intensity of wave fields. Setting $u_{\mathrm{c}}$ be $v_{\mathrm{T}} / v_{\mathrm{r}}$, where $v_{\mathrm{T}}$ is the thermal velocity, and considering the lower bound (70) and 

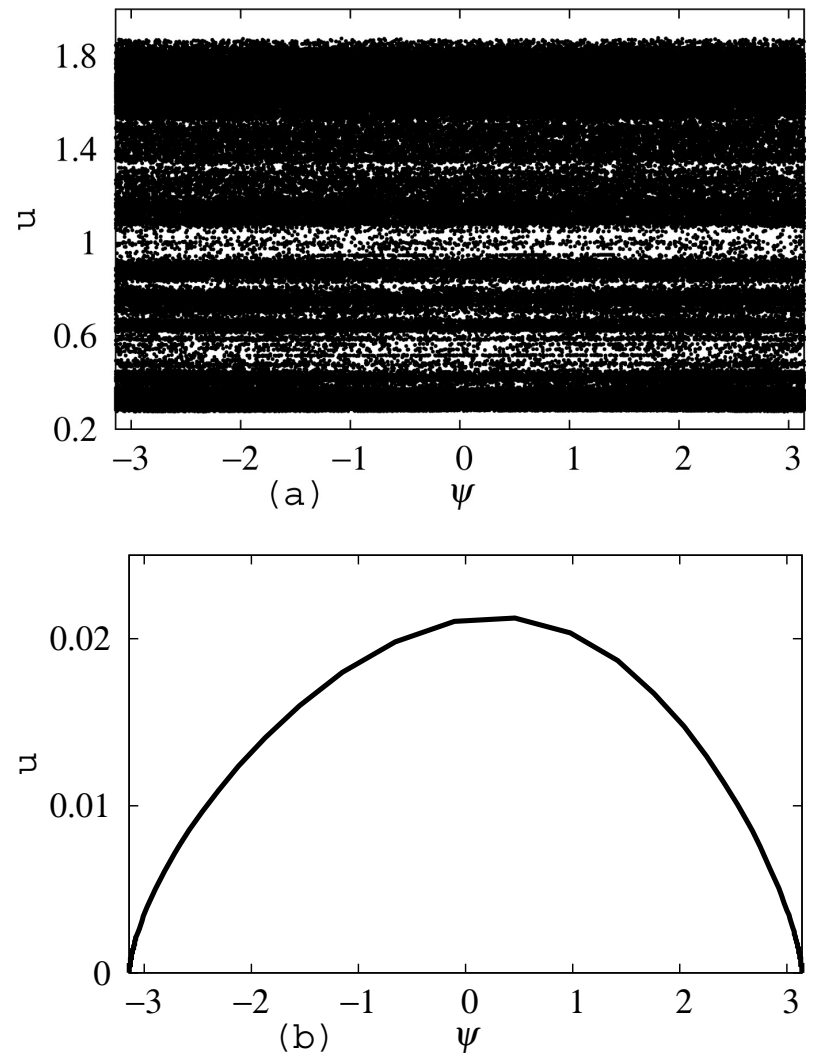

Fig. 4. Phase space for the map $G^{n}$ at $Q=2 \times 10^{-3}$. (a) A single trajectory of length $2 \times 10^{5}$. The initial point $\left(10^{-4}, 3 \times 10^{-1}\right)$. (b) A single trajectory of length $8 \times 10^{3}$. The initial point $\left(10^{-4}\right.$, $\left.10^{-5}\right)$.

$u_{\mathrm{c}}^{2} \ll 1$, we evaluate the available wave intensity,

$P_{\text {th }} \geq 10^{-8} \mathrm{nT}^{2} \mathrm{~Hz}^{-1}, B_{\text {th }} \geq 1 \mathrm{pT}$.

The equations from which the SA arises are usually parameterized by some control parameter, whose variation changes the character of the dynamics. The control parameter $Q$, as a rule, depends directly on the intensity of the wave field. First, we consider the dynamics of $g^{n}$ under conditions when the wave perturbation is larger than that observed. Figure 5 shows the evolution of the system over time at $Q=5 \times 10^{5}$. The picture indicates that the system demonstrates both chaotic and regular dynamics, a so-called chaotic intermittent behavior. This behavior is more complex, exhibiting random transitions between regular and stochastic motion. In this regime, stochastic diffusion governed by the SA is realized through the drift of orbits in phase space and can be expressed in terms of a FPK equation that describes an increment in entropy rather than the diffusion in action (Khazanov et al., 2008b). The phase modification is associated with the appearance of fixed elliptic points, which occur when an initial saddle point of the attractor changes to a stable elliptic point as the control parameter $Q$ increases above the appropriate critical value, $Q_{\mathrm{c}}$. To determine these points,
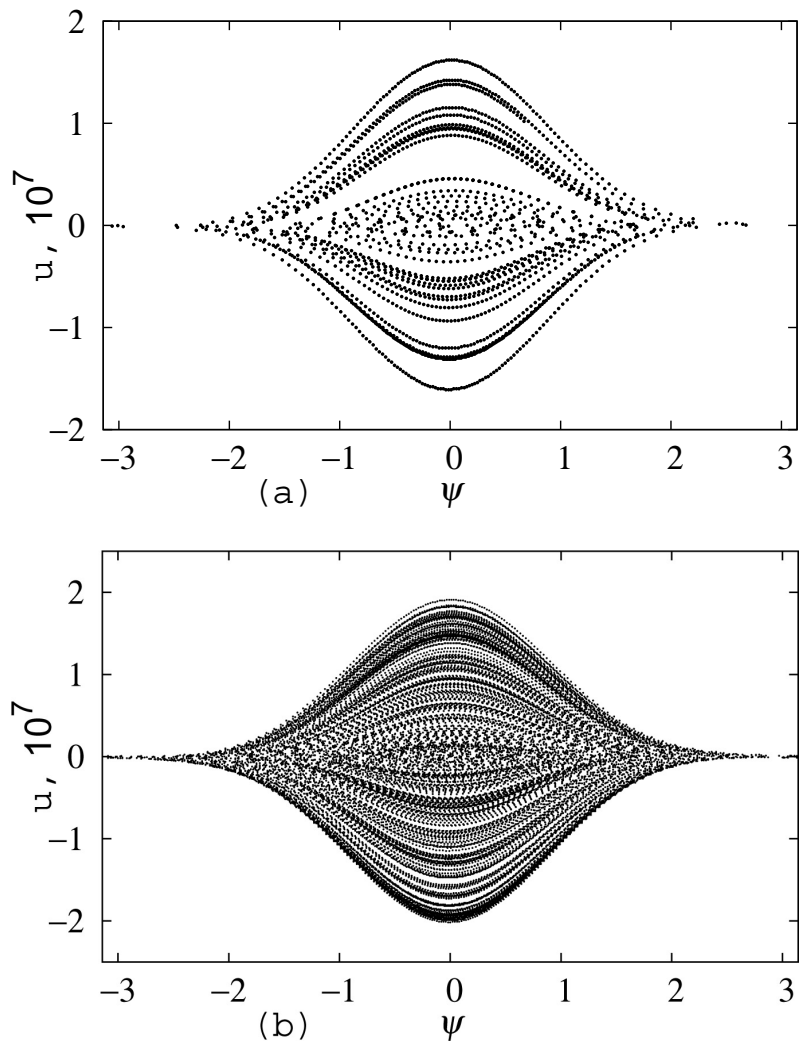

Fig. 5. Intermittent chaotic behavior of map (62) with $Q=5 \times 10^{5}$. (a) $t=2 \times 10^{3}$ and (b) $t=2 \cdot 10^{4}$ iterations of a single initial condition.

we write

$\sin \psi_{0}=0,\left(3 K / 2 u_{0}^{2 / 3}\right)=2 \pi, u_{0}=u_{\mathrm{b}}$,

where $u_{\mathrm{b}}$ is given by expression (68). These equations are satisfied provided $Q$ takes the value

$Q=Q_{\mathrm{c}}, \quad Q_{\mathrm{c}}=(3 K / 4 \pi)^{5 / 2} K^{-1}$.

The test does predict the phase modification rather well. We find this behavior numerically and can give an interpretation in terms of the theory developed by Khazanov et al. (2008b). Substituting $Q_{\mathrm{c}}$ in Eqs. (69) and (74), we obtain $u_{0} \simeq 3 \times 10^{3}$, and therefore $E_{0} \simeq 100 \mathrm{MeV}$. Finally, Eq. (75) yields $Q_{\mathrm{c}} \simeq$ $10^{3}$, which is three orders of magnitude larger than typical values of $Q(\sim 0.5-1)$. The explicit expression for $P_{\mathrm{c}}$, the value of the wave power at which pronounced intermittent dynamics occur, results immediately from Eq. (64):

$P_{\mathrm{c}}=(2 / 9)(3 / 4 \pi)^{5}(\omega T)^{2}\left(B^{2} / \omega\right), P_{\mathrm{c}} \sim 10^{3} \mathrm{nT}^{2} \mathrm{~Hz}^{-1}$.

The results show that the intermittent chaotic motion is possible only in extremely strong $\left(B_{\mathrm{w}} / B \sim 1\right)$ wave fields. Under typical conditions, the long-term evolution of the system is dominated by diffusion induced by stochasticity. 


\subsection{Statistical properties of the dynamics}

The dynamics of the system exhibits a random walk, and all points of the phase trajectory tend to a certain strange attractor (SA) at $n \rightarrow \infty$. The appearance of such an SA represents persistent chaotic motion over a global domain of phase space, as shown in Fig. 6. There the joint probability density $\rho(\psi, u, t)$ is proportional to the number of phase points in the element of phase space. We see that after a few iterations the action distribution remains localized while phase randomization occurs, and that the final distribution is random. Thus, we have good evidence for statistical properties in the region for which the system exhibits chaotic motion. Once the phase variable becomes rapidly varying, the evolution of the coarse-graining function

$w(u, t)=\frac{1}{2 \pi} \int_{-\pi}^{\pi} \rho(\psi, u, t) d \psi$,

obeys the Fokker-Planck-Kolmogorov (FPK) equation for the $u$ variable alone. In this case, the nature of the evolution to the steady state is so-called deterministic diffusion (Lichtenberg and Lieberman, 1992). As the canonical status of the phase variables has been demonstrated above, the distribution function (probability density) $w(u ; t)$ is governed by the FPK equation in the standard form

$\frac{\partial w(u ; t)}{\partial t}=\frac{1}{2} \frac{\partial}{\partial u} D \frac{\partial w}{\partial u}$.

Here $D$ is the conventional diffusion coefficient in phase space,

$D=<\left(u_{n+1}-u_{n}\right)^{2}>T^{-1}=Q^{2} / 2 T$,

where $\left(u_{n+1}-u_{n}\right)$ is substituted from either $g^{n}$ or $G^{n},<\cdot>$ denotes the phase average, and $T$ is the timescale of the problem. The function $w(u, t)$ is a differentiable function supported in $\{U\}$ with the norming

$\int_{u \in\{U\}} w(u, t) d u=1$,

where $\{U\}$ is a range of the variable $u$.

First, by means of $g^{n}$ (or $G^{n}$ ) we calculate the diffusion coefficient and evaluate the characteristic time for redistribution of $u$ over the spectrum

$t_{d} \simeq 2 u_{m}^{2} / D=T\left(2 u_{m} / Q\right)^{2}$.

We exploit the FPK equation with $w(u)$ and its derivative $\partial w / \partial u$ vanishing at the upper and lower boundaries. We introduce the moment $\left.<u^{2}\right\rangle=\int_{-\pi}^{\pi} \mathrm{d} u u^{2} w(u)$, multiply equation (78) by $u^{2}$, and integrate the resulting equation over $u$ to obtain

$\mathrm{d}<u^{2}>/ \mathrm{d} t=D, u \in\left\{U \mid u \leq u_{m}\right\}$.
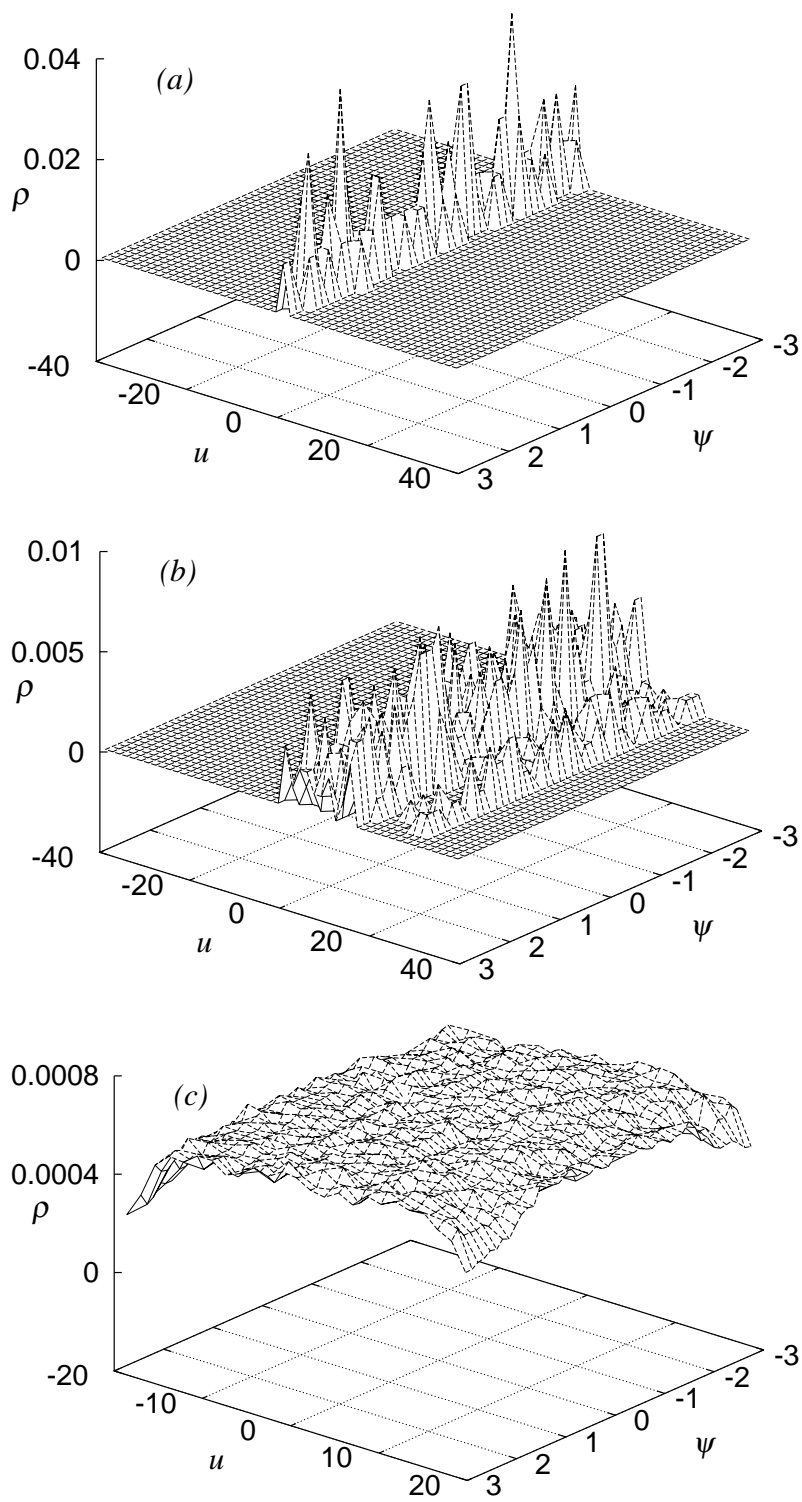

Fig. 6. Joint distribution $\rho(\psi, u)$ computed numerically via $g^{n}$ at several different numbers of steps: (a) $10^{2}$, (b) $10^{3}$, and (c) $2 \times 10^{6}$. The parameters are the same as in Fig. 3a.

The restriction on $u$ is needed because the spectrum is bounded from above. Then, by means of Eq. (60) along with Eq. (72), we calculate by formulas (79) and (81) the diffusion coefficient $D_{u} \approx 1 \mathrm{~s}^{-1}$ and the time of diffusion in $u$, $t_{d} \sim 16 \mathrm{~s}$. For the function $w(u)$ restricted to $\left[u_{\mathrm{c}}, u_{0}\right]$, the invariant distribution can be given by

$w(u)=\left(u_{0}-u_{\mathrm{c}}\right)^{-1}$.

This means that the random variable $u$ is evenly distributed on $\left[u_{\mathrm{c}}, u_{0}\right]$. Now we evaluate the energy distribution function, $w(\varepsilon)$, in the range of non-relativistic energies. Considering $u^{2}=4(\sqrt{e}-1)$, we note that the particle energy $E$ is a specified function of $u$. Thus the measure-preserving 
transformation $w(e) d e=w(u) d u$ determines this problem completely, subject to appropriate boundary conditions. The corresponding solution for $w(e)$ is found to be

$w(e)=w(u) / 2[e(\sqrt{e}-1)]^{1 / 2}, e \in\left(e_{\mathrm{c}}, e_{0}\right)$.

By means of Eq. (84) we find the mean energy $\langle e\rangle \sim 0.5 e_{0}$. This solution relates directly to the behavior of the system near the order-chaos bifurcation transition. Equation (82) may be written as

$\mathrm{d}<v_{t}^{2}>/ \mathrm{d} t=D_{u} v_{\mathrm{r}}^{2}$.

Then considering Eq. (85) we find the rate of change of magnetic moment $\mu, \dot{\mu}=v_{\mathrm{r}}^{2} D_{u} / 2 B$, and the heating rate $\dot{E} \simeq E_{0} / t_{d}\left(\sim 6 \mathrm{keV} \mathrm{s}^{-1}\right)$. This result is nontrivial, because chaotic motion in the $(u, \psi)$-phase space leads to nonadiabatic magnetic moment changes and stochastic heating of plasma particles. The gyroradius is a direct measure of the perpendicular electron velocity. Thus the stochastic heating will be accompanied by a radial drift of particles in space. Indeed, considering the relation $r=v_{t} / \omega_{B}$, via (85), we get the following expression

$\frac{\mathrm{d}<r^{2}>}{\mathrm{d} t}=D_{t}, D_{t}=D_{u} v_{t}^{2} / \omega_{B}^{2},\left(\sim 10^{10} \mathrm{~cm}^{2} \mathrm{~s}^{-1}\right)$,

where $D_{t}$ is the coefficient of collisionless diffusion across the ambient magnetic field.

Now we describe the effects associated with stochastic heating of high-energy particles. Due to one-to-one correspondence, $u=p^{3 / 2}$, and using $d p=2 d u / 3 p^{1 / 2}$, one obtains the coefficient of diffusion in $p, D_{p}=4 D_{u} / 9 p$. Then the measure-preserving transformation $w_{p}=w_{u}|\partial u / \partial p|$ results in the probability density in $p, w_{p}=3 \sqrt{p} / 2 p_{\mathrm{b}}^{3 / 2}$. There is an important relationship, $w_{p}^{2} D_{p}=w_{u}^{2} D_{u}$. Furthermore, one can easily prove the invariant relation

$w_{v}^{2} D_{v}=w_{u}^{2} D_{u}=$ inv., inv. $=1 / 2 t_{d}$

takes place for any $v(u)$, and depends smoothly on $u$, the coordinate on the SA, whose distribution function $w_{v}=w(v)$ relates with $w_{u}$ through a measure-preserving transformation, with $D_{v}$ given by a quadratic form of its variation. We see that the invariant is given by the rate of diffusion on the SA. We come to know as well that the FPK equation for $w(v, t)$ can be written in divergent form

$\frac{\partial w(v, t)}{\partial t}=\frac{\partial}{\partial v} J(v), J(v)=\frac{1}{2}\left(D_{v} \frac{\partial}{\partial v}+d_{v}\right) w(v, t)$,

where the transport coefficients are given by

$D_{v}=D_{u}(\partial v / \partial u)^{2}, d_{v}=\frac{1}{2} D_{u} \partial / \partial v(\partial v / \partial u)^{2}$.

The above equations have important consequences in determining the heating rate. Then, introducing the particle energy distribution $w(e, t)$, and taking into account the invariant of motion and the relation $e=E / m$ and $E=$ $\sqrt{m^{2}+p_{z}^{2}+2 m \omega_{B} I}$, we find, after appropriate transformations, that the following equation for $w(e, t)$ results in

$$
\frac{\partial w(e, t)}{\partial t}=\frac{\partial}{\partial e} J(e), J(e)=\frac{D_{u}}{2}\left(\frac{4}{9 e} \frac{\partial}{\partial e}-\frac{2}{9 e^{2}}\right) w(e, t) .
$$

Then it may be possible to find the heating rate

$\frac{\mathrm{d} E}{\mathrm{~d} t} \simeq \frac{8}{9}\left(\frac{e_{\mathrm{b}}}{e}\right)^{2} \cdot \frac{E_{\mathrm{b}}}{t_{d}}, t_{d}=\left(\frac{4 e_{\mathrm{b}}^{3}}{Q^{2}}\right) T$,

where $t_{d}$ is the relaxation time to the steady-state distribution. It is worth noting that the injection mechanism at low energies, which may be intimately related to particle heating is the important aspect of space physics. Since the rate of electron cyclotron resonance heating is proportional to $E^{-2}$ this is, indeed the case. We put the characteristic values of these quantities at typical conditions, $\omega_{B} / \omega=2, \omega_{B} / 2 \pi=$ $8 \times 10^{3} \mathrm{~Hz}, P=10^{-4} \mathrm{nT}^{2} \mathrm{~Hz}^{-1}, B=300 \mathrm{nT}, T=2 \times 10^{-1} \mathrm{~s}$, to determine, with the help of Eqs. (69) and (90), the upper boundary energy spectrum $E_{\mathrm{b}} \approx 8 \mathrm{MeV}$, the time of diffusion $t_{d} \approx 2 \times 10^{3} \mathrm{~s}$, and the heating rate $\dot{E} \approx 4 \mathrm{keV} \mathrm{s}^{-1}$, which are all in reasonable agreement with experimental data (Selesnick et al., 1997; Selesnick, 2006). These results lead to two important observations:

1. Nonlinear electron acceleration by a whistler wave is always a stochastic process when the wave power of the wave exceeds the threshold value given by Eq. (73).

2. In this case the related quantities have the following dependencies on the wave power,

$$
E_{\mathrm{b}} \propto P^{1 / 5}, t_{d} \propto P^{-2 / 5}, \dot{E} \propto P^{3 / 5},
$$

with the power law indices determined by the type of nonlinearity in the phase advance equation.

Bursty relativistic electron precipitation (REP) events during geomagnetic storms appears to be the most striking phenomenon in the dynamics of the Earth's radiation belt. The chorus-electron coupling has been closely identified with REP events (Green et al., 2004; O'Brien et al., 2004), and Khazanov et al. (2011) have shown that the process of stochastic electron heating followed by the pitch-angle scattering is actually a viable mechanism for these REP events. The authors have investigated the problem, finding, in particular, that the energy flux in the Earth's atmosphere is directly proportional to the heating rate of a single electron. That is why the enhancement of the flux is often observed during the magnetospheric storm. The result (91) is telling in the Conclusions. 


\subsection{Adaptability of the Fokker-Planck-Kolmogorov equation}

We have seen that diffusion on a strange attractor (SA) is described by a Fokker-Planck-Kolmogorov (FPK) equation for the $u$ variable alone, where $u$ is a canonical variable on the SA, proper for a certain physical situation. The FPK equation to which we refer indicates that an initial $\delta$ function distribution diffuses to a random distribution. Any measure-preserving transformation from $u$ to some new variable $v(u)$ has been shown to preserve the invariant relation $w^{2}(u) D(u)=1 / 2 t_{d}$, with $u$ replaced by $v$, indicating the universal nature of the diffusion process on any SA. In this case, the distribution $f(v)$ can be found by a direct transformation $f(v)=f(u) d u / d v$ without solving a relative FPK equation. However, if we are, for example, concerned with the evolution of the energy spectrum, we should use the FPK in divergent form.

Quasi-linear theory (QLT) does predict the long-time evolution of RB electrons on the basis of an FPK equation. However, in the standard formulation (Lyons and Williams, 1984; Summers et al., 2007), the wave field of whistlers is treated as random, broadband and phase-incoherent. Thus, this formalism can not be directly applied to the problem of electron motion in the regular wave field of chorus. In the nonlinear approach, we apply just the same FPK equation in which phase averaging is made due to dynamics itself, and all time means of observable functions are equal to the phase space means. This approach corresponds to the standard FPK description in statistical mechanics and facilitates the applicability of kinetic models in which the RB dynamics is simulated (Khazanov, 2011). As it is known, QLTs are based on some linearized equations of motion, and the characteristic time of diffusion in energy is given by $t_{d}=E^{2} / D$ that depends on the wave power $P$ as $P^{-1}$ in certain energy ranges. In the nonlinear theory (NLT), the rate of diffusion hinges on the type of interaction, for instance, it obeys the scaling $t_{d} \sim P^{-2 / 5}$ for chorus-RB electron interaction. Shown in Fig. 7 is the time of diffusion as a function of wave power. Since the inequality $t_{d} / T \gg 1$ is a condition for applying the FPK description, these dependencies have important consequences in determining the range of values of wave power for which the FPK formalism is valid. It is just the same thing to evaluate the permissible values of chorus wave fields, $P_{1}, P_{2}$ and susceptible to FPK description, in QLT and NLT, respectively. Here, $P_{1}$ and $P_{2}$ are determined by Eqs. (73) and (76), respectively. They yield $P_{1} / P_{0}=10^{-4}$, and $P_{2} / P_{0}=10^{7}$, where $P_{0}=10^{-4} \mathrm{nT}^{2} \mathrm{~Hz}^{-1}$ is the typical value of wave power (Santolik et al., 2003).

Although it is normally thought that the results from simple problems give qualitative predictions of the behavior of more complicated physical systems, many questions concerning the dynamics of the system still exist. A particularly interesting aspect concerns the effects resulting from the resonance interaction with the quasi-coherent whistler waves.

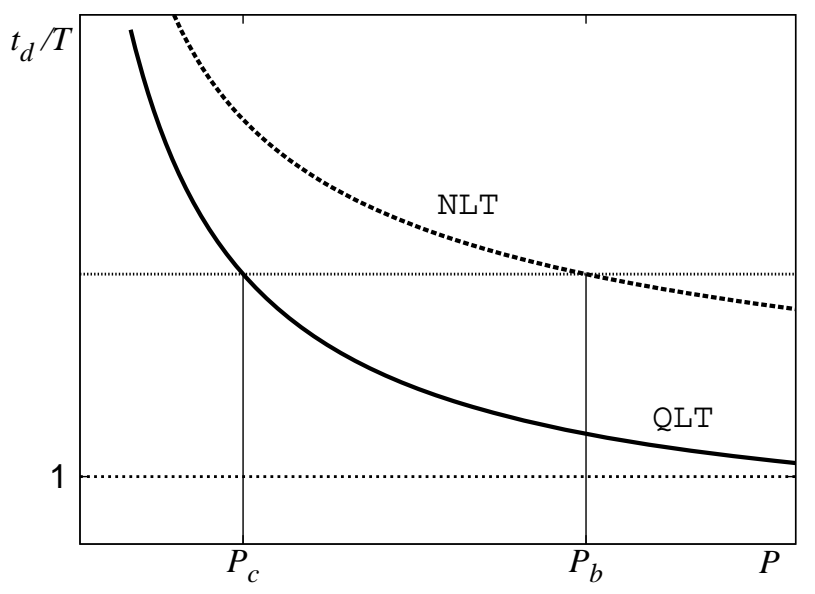

Fig. 7. Time of diffusion as a function of wave power. Here $P_{1}$ and $P_{2}$ are the critical wave powers for the applicability of the FPK equation in QLT and NLT, respectively.

The effect of a magnetic mirror in modifying the motion can also play an essential role in certain physical situations.

Such a physical situation can emerge when RB electrons interact with the so-called quasi-coherent chorus, whose frequency spectrum contains low-intensity isotropic white noise (Tsurutani et al., 2011). The physical explanation for these wave events is quite clear. These are likely to relate to the irregular nature of the Earth's ring current injections, which generate chorus (Liemohn, 2006).

The level of background noise is relatively small; consequently, this effect can exhibit in the equations of motion only as the fluctuations of parameters $\omega_{B}$ and $Q$. Thus the control parameter is a function of the magnitude of wave field $b$, and therefore $Q \rightarrow Q(1+\xi), \omega_{B} \rightarrow \omega_{B}(1+\xi)$. Here, $\xi=\delta b / b$ is the random variable having a Gaussian probability density

$p(\xi)=\frac{1}{\sqrt{2 \pi} \sigma} \exp \left(-\xi^{2} / 2 \sigma^{2}\right)$,

the mean-square value of which is $\left\langle\xi_{n} \xi_{n^{\prime}}\right\rangle=\sigma^{2} \delta_{n n^{\prime}}$, where $\sigma^{2}$ is the intensity of noise, and $\delta$ is the Kronecker delta.

In order to understand how the extrinsic noise modifies dynamics, we consider its influence on motion separately. Thus, we include the additional noise in $G^{n}$ only in the phase advance equation, to obtain the closed pair of nonlinear stochastic equations

$$
\begin{aligned}
& u_{n+1}=u_{n}+Q \sin \psi_{n}, \\
& \psi_{n+1}=\psi_{n}+\xi_{n}+(1 / 4) \omega T u_{n+1}^{2} \bmod 2 \pi,
\end{aligned}
$$

where the term $\xi_{n}$ plays the role of a weak stochastic force.

Numeral investigation of a related quantity, the correlation function $C(k)$,

$$
C(k)=(1 / N) \sum_{n \in N} \psi(n) \psi(n+k),
$$


where $k$ is the step lag, gives the results shown in Fig. 8. The motion has been shown to be $\delta$-correlated, with $C(k)=0$ for $k \neq 0$; this represents a very strong chaotic property, i.e., a complete decorrelation of the motion in one mapping period. Note that FPK description is valid only when the function $C(k)$ falls off rapidly with the number of mapping iterations. The effect of extrinsic noise manifests itself in the FPK equation as an additional term in the coefficient of diffusion, $D / D_{u}=1 \sigma^{2} / Q^{2}$. This effect does not significantly affect diffusion induced by stochasticity, due to the global stability of the SA.

The situation is quite different for ion cyclotron resonance heating (ICRH) (Lichtenberg and Lieberman, 1992). In this case the dynamics is intrinsically degenerate, and the nonlinearity arises from the finite gyroradius, which leads to a spectrum of modes in the motion, and the term proportional to $Q$ occurs in the phase advance equation. This method has been used by the authors in conjunction with the problem of ICRH at the front of a shock (Khazanov et al., 2010).

Now we want to study how the Earth's magnetic mirror effects change the dynamics of $G^{n}$ and how much impact it has on the statistical properties and long-term evolution of the system.

The drift equations for particle motion in a current-free field are given by Schmidt (1979):

$$
\begin{aligned}
\boldsymbol{v}_{\mathrm{d}} & =v_{\|} \boldsymbol{B} / B+[\boldsymbol{F}, \boldsymbol{B}] / B^{2}, F=-\left(\mu+m v_{\|}^{2} / B\right), \\
\mathrm{d} v_{\|} / \mathrm{d} t & =-(\mu / m) \partial B / \partial s,
\end{aligned}
$$

where $v_{\mathrm{d}}$ is the drift velocity, $\boldsymbol{F}$ the free force averaged over a gyro-orbit, $\mu$ the magnetic moment, and $s$ the coordinate along the field line.

In the drift approximation, $\mu$ is an adiabatic invariant, which is assumed to be well conserved. Since the energy of a charged particle gyrating in the Earth's magnetic mirror is strictly conserved, $v_{\|}$is related to the constants of the motion $\mu$ and $E$ through the relation $v_{\|}=\sqrt{2(E-\mu B) / m}$, which is easily calculated for a given function $B$. In the equations of motion, describing the resonant interaction with high-frequency wave fields, the forces given by Eq. (93) average to zero, as do all other off-resonant terms. All the same, residual effects are always present, which lead to the usual distortions of the phase plane near resonances of the principal frequencies, and can be physically associated with the loss of phase coherence from one passage along the field line. To show how this occurs, we first write Eq. (10) as $y=y_{d}+r \cos \theta$, where $y_{d}$ is the guiding center position of the gyrating particle. Then, this dependence in the $\psi$ variable gives rise to the term $k_{t} y_{d}$, and as a consequence, the term $\left\{k_{t} v_{\mathrm{d}} T\right\}_{n}-\left\{k_{t} v_{\mathrm{d}} T\right\}_{n+1}=\left\{k_{t} v_{\mathrm{d}} T\right\}$, where $\{\cdot\}$ is the fractional part, occurs in the phase advance equation. We can eliminate this term by a shift of $z$ or $t$; however, small asymmetries or time variations in the magnetic field exist, which allow stochastic diffusion to occur. If these variations are random, we would write an independent equation $\left\{k_{t} v_{\mathrm{d}} T\right\}=\xi_{n}$,

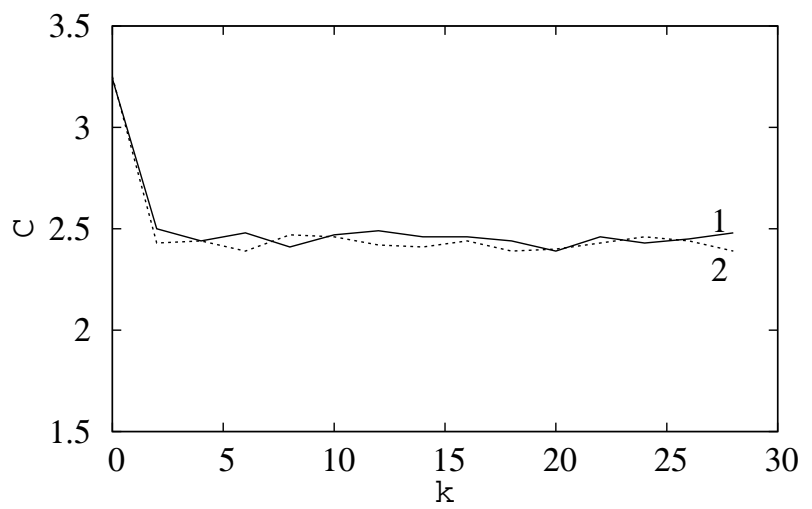

Fig. 8. Dependence of the correlation function $C(k)$ on $k$, corresponding (1) to the map $G^{n}$ without noise and (2) with noise (92) for calculations over 2000 points.

where $\xi_{n}$ is a random variable. At last, assuming that these fluctuations are white noise, we arrive at equations of motion that are similar to the map (92). Hence, the system generates diffusion analogous to that described above. Some numerical experiments seem to verify that behavior (Faith et al., 1997; Roth et al., 1999; Zheng et al., 2012, 2013). It is obvious that the effect of bouncing on the dynamics may be investigated in just the same way.

It should be noted that the term $\xi_{n}$ in the system acts without significantly changing its statistical properties; moreover, numerical investigations obviously reveal that the SA remains in the sense such that we can't tell the SA of $G^{n}$ from that of system (92) by whichever sign. However, if we remove the nonlinear term from the phase advance equation, then the term dominates the behavior of the system fully.

Now, we examine the problem of energization of RB electrons subject to resonant coupling with oblique largeamplitude whistler waves. The existence of such waves is confirmed by direct measurements (Cattell et al., 2008; Cully et al., 2008; Kellogg et al., 2011; Kersten et al., 2011; Wilson et al., 2011). In our calculation, we will rely on the relevant experimental data of Cattell et al. (2008) and Wilson et al. (2011). These waves have a broad range of wave normal angles $\vartheta$ with respect to the magnetic field $\left(20-60^{\circ}\right)$ and large peak-to-peak amplitudes $E_{1} \sim(100-200) \mathrm{mVm}^{-1}$ with $B_{t} \sim 1 \mathrm{nT}, E_{t} \sim 100 \mathrm{mVm}^{-1}$. The timescale of the envelope is typically $T \sim 0.2 \mathrm{~s}$, and the mean value of the frequency at peak wave power is $(0.2-0.25) \omega_{B}(\sim 1.5 \mathrm{kHz})$. We have used this data, as well as the dispersion relation $\omega=\omega_{B}\left(k^{2} / \omega_{\mathrm{p}}^{2}\right) \cos \vartheta\left(\right.$ Stix, 1992) where $\omega_{\mathrm{p}}$ is the electron plasma frequency $\left(\sim 1.1 \times 10^{5} \mathrm{~s}^{-1}\right)$, to estimate the wavenumber $k \sim 2 \times 10^{-4} \mathrm{~m}^{-1}$ and group velocity $v_{\mathrm{g}} \sim 5 \times$ $10^{7} \mathrm{~m} \mathrm{~s}^{-1}$.

From Hamiltonian (18) we see that the amplitude of the interaction terms is proportional to Bessel functions, and the Bessel coefficients $J_{s}\left(k_{t} r\right)$ give the fall-off of the 
Fourier amplitudes. Thus we will examine only the resonance of driving with the fundamental of gyromotion, $k_{z} p_{z} / E+$ $\omega_{B} m / E-\omega=0$. Then, because the Bessel functions tend to zero as $1 / \sqrt{k_{t} r}$ at $k_{t} r \rightarrow \infty$, we have to choose $k_{t} r \approx 1-3$, which corresponds to the values of $J_{1}(\cdot)$ near a maximum in the Bessel function. This choice automatically imposes certain restrictions on the energy spectrum of particles. Indeed, taking into account the invariant of motion (25), and the relations $k_{t} r \sim(1-3), \omega_{B} / k_{z} \sim 1, r=\sqrt{2 m \omega_{B} I} / m \omega_{B}$, we find, without much difficulty, the range of particle energies, in which the wave-particle interaction is most effective, $\left(k_{t} / k_{z}\right) \sqrt{2 E / m} \sim(1-3), E<10 \mathrm{MeV}$.

First we will study the problem provided the condition $B_{t} / B \gg\left(J_{1}(\cdot) U_{0} / J_{1}^{\prime} m\right) \sqrt{E / m}$ holds. By integrating Eqs. (21)-(23) along with invariant of motion (25), we derive, in the usual way, a map that is similar to the $g^{n}$ map describing the chorus-electron interaction, except that the control parameter contains the term $J_{1}^{\prime}\left(k_{t} r\right)(\sim 0.1)$. This similarity means that the given dynamical system manifests itself in just the same way as the $g^{n}$ map; as a consequence, the heating rate is governed by the same Eq. (90). However, the intensity of coupling, in this case, is determined by the magnitude of the wave field multiplied by the factor $J_{1}^{\prime}(\cdot)$. It stands to reason that the expression (91) remains the same, after appropriate modifications, $\sqrt{P} \rightarrow \sqrt{P} J_{1}^{\prime}(\cdot)$. So these waves are about four times larger in magnitude than those measured by SAMPEX (The Solar Anomalous and Magnetospheric Particle Explorer), and the heating rate of the process is comparable to that caused by chorus-electron coupling.

Let the dynamics be dominated by interaction of the particle with the electrostatic (ES) component of a wave. Taking the wave field to be in the form of a space-like (SL) wave packet (54), we convert the equations of motion into the map,

$u_{n+1}=u_{n}+Q \sin \psi_{n}, \psi_{n+1}=\psi_{n}+1 / u_{n+1}, \quad \bmod 2 \pi$,

where the variable $u$ and the control parameter $Q$ are defined by the relations,

$u=(E / m \omega T), Q=J_{1}(\cdot) U_{0} / m \sqrt{N}$,

and the spectral characteristic $N$ and the ES potential are given by

$N=\Delta \nu T, U_{0}=E_{0}^{l} / k_{z}$.

The map (94) belongs to the class of Fermi mappings, therefore this map possesses an SA (Lichtenberg and Lieberman, 1992). Shown in Fig. 9 is the SA of the system, whose upper bound is defined by the equations

$u_{\mathrm{b}}=\sqrt{Q}, E_{\mathrm{b}}=\omega T \sqrt{Q} m$,

which is borne out numerically.

The invariant distribution on the SA is a trivial constant, and the time of diffusion in energy is found to be

$t_{\mathrm{d}}=(4 / Q) T$.

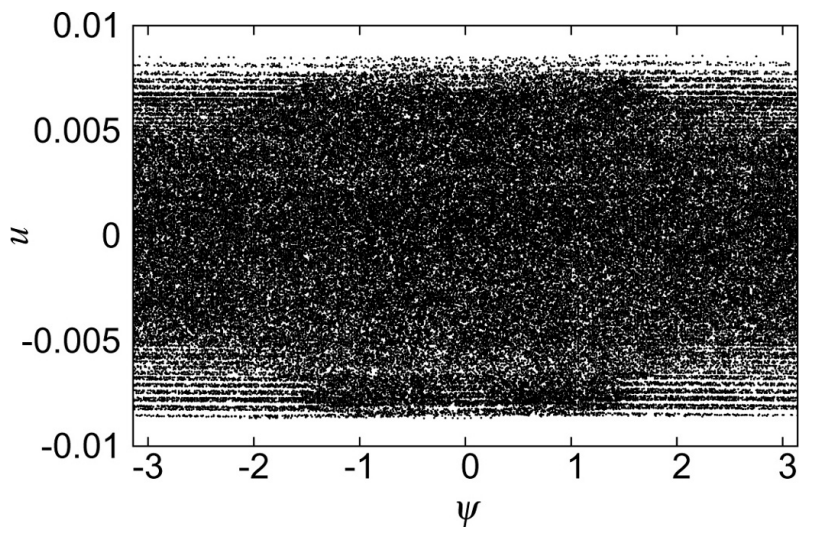

Fig. 9. Phase space $u-\psi$ for the map (94) at $Q=0.98 \times 10^{-4}$.

We see the extent and rate of heating at this wave-particle interaction are proportional to $U_{0} J_{1}\left(k_{t} r\right)$, just as it is expected. We extract from Eqs. (94) and (98) the following estimates of $E_{\mathrm{b}} \simeq 5 \mathrm{MeV}$ and $t_{\mathrm{d}} \simeq 10^{4} \mathrm{~s}$. We have seen that generic, nonlinear behavior in the interaction with oblique waves restrict the extent and rate of particle heating, which, to a certain extent, accounts for the nature of wave-particle interaction.

Electron acceleration in the radiation belts is a wellestablished fact. Whistler-RB electron coupling ranks among the most important accelerate mechanisms that are likely to play a major role in maintaining the entire pool of RB electrons. Acceleration of RB electrons, which are driven by whistlers up to relativistic energies, can proceed in the very short time of $\sim 1 \mathrm{~h}$ or less, and manifest itself in bursty relativistic electron precipitation events. We have used topological arguments to determine the value of the control parameter at which the dynamical system exhibits chaotic motion, in the form of a strange attractor. In this case, every particle can explore the entire phase space energetically accessible to it; as a result, the upper bound of the strange attractor can be put on a one-to-one correspondence with the upper boundary of an energy spectrum whose value depends parametrically on the spectral power of the wave. The chaotic motion on the strange attractor is ergodic with mixing, and as a consequence the evolution of the distribution function and all means obeys a FPK equation.

As it appears from our model, one thing is certain: the selfconsistent nonlinear approach to the RB electron dynamics offers far more perspective than the QLT. There is every reason to believe that an FPK description has turned out to be a true one, after a fashion. The nonlinear approach suggests that it may be possible to obtain theoretically a kind of scaling law for the rate of diffusion that would be applicable to most whistler wave events. Thus, studying the model of nonlinear diffusion can lead to important results, both from the point of view of interpreting experimental data and of obtaining reliable information from the approach. 


\section{Stochastic acceleration of cosmic-ray electrons}

It is well known that high-energy particles observed in cosmic rays can be regarded as tails of the particle distribution in space plasmas. The energy relation (the energy per unit volume of space plasma is approximately equal to the energy per unit volume of cosmic rays) shows that collective processes occurring in the plasma can play an important role in the evolution of the energy spectra of cosmic rays. In this connection, it becomes relevant to determine how the energy should be redistributed in cosmic rays so that the energy spectra could contain particles with very high energies (Kaplan and Tsytovich, 1973; Ginzburg, 1989). Supernova remnant shocks are thought to be the primary source of cosmic rays, because supernova remnants (SNRs) are able to provide the energy necessary to maintain the observed cosmic ray density. Synchrotron emission from SNRs indicates the presence of $100 \mathrm{TeV}$ electrons, and inverse Compton scattering of background photons by ultrarelativistic (UR) electrons is a very plausible explanation for $\mathrm{TeV} \gamma$-ray emission from young SNRs (Reynolds, 2001; Treumann and Terasawa, 2001; Vink, 2004; Abdo et al., 2009).

The current description of cosmic ray acceleration up to UR energies is the well-known first-order Fermi acceleration at the SNR shock front (Bell, 1978; Blandford and Ostriker, 1978). In the first-order Fermi model, wave turbulence makes the particle momenta isotropic, thus particles can cross the shock front many times. Simulations show that the efficiency of the mechanism depends on the spectra and amplitudes of MHD fluctuations, and their fluctuation amplitudes, and relativistic particle distributions are a natural consequence of the stochastic acceleration by turbulent plasma waves. As the effect of energy loss through synchrotron emission is not included in the model, the gradual high-energy cutoff has been attributed to the balance between the acceleration and escape processes, which leads to a steady-state distribution (Liu et al., 2008; Muranushi and Inutsuka, 2009). In addition to this, it should be noted, the wave-particle interactions in the model are treated in the linear approximation.

There is also another treatment of the problem relating Hamiltonian chaos to nonlinear interactions of particles with plasma waves (Sagdeev et al., 1988; Horton and Ichikawa, 1996). A dynamical system that can be represented by a measure-preserving map and that illustrates the nature of stochasticity was proposed by Fermi as a model for cosmic ray acceleration. There are, as a matter of fact, various versions of this model, all characterized by a phase advance equation that is inversely proportional to the velocity. The review of the class of Fermi maps was made by Lichtenberg and Lieberman (1992). Another important model is the relativistic standard map (RSM), which was independently proposed by Chernikov et al. (1989) and Nomura et al. (1992). The RSM is the relativistic generalization of the standard map (SM), introduced by Chirikov (1979). The standard map is well known for application to a wide class of prob- lems, including the confinement of particles in fusion devices, radio-frequency heating, acceleration, and heating of particles by nonlinear wave packets (Lichtenberg and Lieberman, 1992). Thus, it was natural to study in terms of the RSM how relativistic effects modify the nonlinear motion described by the SM. It is worth noting that these investigations of RSM provide us with a more profound knowledge of many general properties of relativistic chaotic systems. A possible mechanism for strong heating was proposed by Papadopoulos, and the first simulation was performed by Cargill and Papadopoulos (1988). It was shown that collisionless shock waves propagating away from a supernova may be directly responsible for the $10 \mathrm{keV} \mathrm{X}$-ray emission seen in SNRs. A sequence of plasma instabilities between the reflected ions and the background electrons at the foot of the shock front can give rise to rapid anomalous heating of electrons. A somewhat different approach to the problem was taken by Dieckmann et al. (2004). A self-consistent simulation was performed by these authors to show that ions reflected from SNR shocks can excite large-amplitude electrostatic waves through the beam-plasma instability. Another mechanism that has been investigated to explain the acceleration process includes the nonlinear coupling of the beam of cosmic rays escaping away from a supernova, with background Langmuir waves. Estimates of the rate of instability from this type of interaction were made by Ginzburg (1989), who found that the characteristic time of the instability would be about a few years.

As a consequence of this, chaotic dynamics of relativistic electrons is of particular interest. Thus, the nonlinear interaction of high-energy electrons with an extraordinary electromagnetic wave propagating across a given magnetic field was investigated by Zaslavsky et al. (1987). They estimated the rate of the diffusion and found it to be sufficient to accelerate an electron up to UR energies. Chaotic dynamics of relativistic electrons in the spectrum of Langmuir waves has been considered by Klimov and Tel'nikhin (1995) in terms of a map given by the closed pair of nonlinear difference equations. More complete calculations, including the gyroresonance effect were made by Nagornykh and Tel'nikhin (2003) who showed the role of the gyromotion in randomizing the phase of particles. Finally, Krotov and Tel'nikhin (1998) have shown that the stochastic heating of particles by the nonlinear Langmuir wave packet in space plasmas can be regarded as a possible mechanism for the formation of the energy spectrum of cosmic rays.

\subsection{Stochastic surfing electron acceleration at Galactic shocks}

Besides the Fermi model for cosmic ray acceleration, there is another possible mechanism for the generation of cosmic rays proposed by Teller (1971). In this approach, the shock wave emerging at a supernova explosion is capable of accelerating cosmic ions with the spectrum which quiet good 
corresponds to the empiric spectrum. However, this spectrum of escaping relativistic ions turns out to be unstable in regard to some plasma instabilities. The self-consistent simulation of Dieckmann et al. (2004) showed that such a beam of relativistic ions can induce a collisionless shock. This shock arises as a consequence of strong nonlinear upper-hybrid (UH) solitary waves which are driven via the beam instability by the ion beam. In this scenario, typical for collisionless shock generation (Artsimovich and Sagdeev, 1979), the shock front is formed by the dissipative process caused by particle heating, and the tail of the wave manifests itself as UH wave perturbations in the foot of the quasi-perpendicular shock. The dispersion law of the electrostatic UH wave (its wave potential is about $1 \mathrm{mc}^{2}$ ) at the foot of shock front is

$\omega=k v_{\mathrm{f}}, v_{\mathrm{f}} \leq v_{\mathrm{b}}, \omega=\sqrt{\omega_{B}^{2}+\omega_{\mathrm{p}}^{2}}$,

the dispersion law of which is a typical condition for plasma modes induced by a beam (Artsimovich and Sagdeev, 1979), where $\omega_{\mathrm{p}}$ is the electron plasma frequency, and $v_{\mathrm{f}}, v_{\mathrm{b}}$ are the speed of the front and beam respectively. In this situation electrons can be accelerated up to relativistic energies through the mechanism of surfing (Sagdeev et al., 1988). The model of surfing is in general similar to the Fermi-Ulam model (Lichtenberg and Lieberman, 1992) of acceleration in which the fixed wall plays the role of Lorentz force. This force doesn't change the particle energy and results only in the drift of particles along the front (look at the picture in Fig. 10). The way the electrons gain energy is by picking up wave field energy in multiple encounters with the shock front (Sagdeev et al., 1988).

Taking the original potential of the wave to be of the form

$\varphi(y, t)=\varphi_{0} \cos (k y-\omega t)$,

then Eq. (22) can be written as

$$
\begin{aligned}
& \dot{I}=\sum_{n} \varphi_{0} n J_{n}(k r) \sin (n \theta-\omega t), \dot{\theta}=\omega_{B} m / E, \\
& E=\sqrt{m^{2}+2 m \omega_{B} I}, \quad \psi=n \theta-\omega t .
\end{aligned}
$$

In this case electron cyclotron resonance heating (ECRH) is accomplished by resonance between the gyrofrequency and the UH wave,

$\dot{\psi}=s \omega_{B} m / E_{\mathrm{r}}-\omega=0, \quad s \in Z_{\mathrm{s}} \subset Z$,

which is satisfied for a series of $s$-values for particles of different energies.

To begin with, let us consider the dynamics in a single mode. Choosing a particle resonance $s=l$, we can write Eqs. (101)-(102) as

$\dot{I}=\varphi_{0} l J_{l}(k r) \sin (\psi), \quad \dot{\psi}=l \omega_{B} m / E-\omega$.

Linearizing with Eqs. (104) in the vicinity of the exact resonance, putting of quantities at the resonance, we obtain the

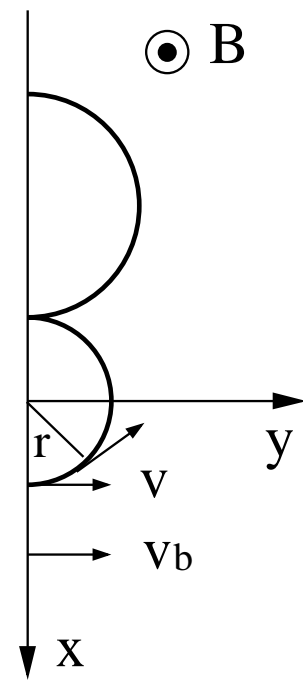

Fig. 10. Particle trajectory at the front of a shock.

equation of phase oscillations

$$
\begin{aligned}
\ddot{\psi}+\omega_{\mathrm{b}}^{2} \sin \psi & =0, \omega_{\mathrm{b}}=\frac{\omega_{B}}{e} \sqrt{\left(\frac{\omega}{\omega_{B}}\right)^{5 / 3} e^{2 / 3} U_{0}}, \\
e & =E / m, \quad U_{0}=\varphi_{0} / m,
\end{aligned}
$$

where $\omega_{\mathrm{b}}$ is the bounce frequency in the wave field. In writing (105) we have taken advantage of the dispersion law (99), the relation $(k r)_{\mathrm{r}}=l$, and used the asymptotic expression of the Bessel function $J_{l}(l) \approx 1 / l^{1 / 3}$, which is valid for $l \gg 1$. By requiring $\omega_{\mathrm{b}} \geq \delta \omega$, where $\delta \omega=\omega_{B} / e$ is the spacing between adjacent modes, we obtain the overlap condition

$e_{\mathrm{c}} \geq\left(\omega_{B} / \omega\right)^{5 / 3} / U_{0}^{3 / 2}$,

which indicates the value of wave field at which electrons with energy $e$ can be accelerated in random manner. At last, we find from above the characteristic time of the variation of $I$ and $\psi$ is of order $2 \pi / \omega_{\mathrm{b}} \sim\left(2 \pi / \omega_{B}\right) e$, which essentially exceeds one period $2 \pi / \omega$. The small parameter $\varepsilon=$ $\omega_{B} / \omega e \approx 1 / s$ serves as the condition for the applicability of the adiabatic approach, developed by the authors (Khazanov et al., 2010).

The condition of adiabaticity, $\dot{\psi} \ll \omega \psi, \dot{I} \ll \omega I$ allows us to write Eqs. (101)-(102) in the form

$\dot{\psi}=s \omega_{B} e^{-1}-\omega, \dot{I}=2 s J_{\mathrm{s}}(s) \varphi_{0} \sin \psi T \sum \delta(t-n T)$,

$T=2 \pi e / \omega_{B}$.

We have carried out the following transformation here,

$\sum \varphi_{0} n J_{n}(\cdot) e^{i n \theta-i \omega t}=\varphi_{0} s J_{S}(s) e^{i s \theta-i \omega t} \sum_{n \neq s} e^{i n \omega_{B} t / e}$,

employed the identity $\sum e^{i n \omega_{B} t / e}=T \sum_{n} \delta(t-n T)$, and taken into account that the wave-particle collisions occur 
twice during one gyroperiod. After integrating, Eqs. (107) reduce to a map

$e_{n+1}=e_{n}+Q e_{n}^{2 / 3} \sin \psi_{n}, Q=2\left(\omega / \omega_{B}\right)^{2 / 3} U_{0}$,

$\psi_{n+1}=\psi_{n}+2 \pi\left(\omega / \omega_{B}\right) e_{n+1} \bmod 2 \pi$,

where $s=\left(\omega / \omega_{B}\right) e(\gg 1), \quad J_{\mathrm{S}}(s) \approx 1 / 2 \pi s^{1 / 3}$ have been used.

Now we have to obtain the equations of motion under conditions of strong energy losses due to synchrotron emission and inverse Compton scattering, the rate of which is given by

$\dot{e}=-\beta_{\mathrm{s}} e^{2}$,

where $\beta_{\mathrm{s}} \approx 0.5 \times 10^{-19} \mathrm{~s}^{-1}$ at $B=3 \mu \mathrm{G}$ (Ginzburg, 1989). Calculating the loss of energy over the gyroperiod yields

$\Delta e=-\alpha e^{3}, \alpha=\beta_{\mathrm{s}} / v_{B}, v_{B}=\omega_{B} / 2 \pi$,

which is valid if $\Delta e / e \ll 1, e<10^{10}$. Then we combine Eq. (108) and Eq. (109), to obtain a dissipative map

$$
\begin{aligned}
e_{n+1} & =e_{n}\left(1-\alpha e_{n}^{2}\right)+Q e_{n}^{2 / 3} \sin \psi_{n}, \\
\Delta \psi & =2 \pi \frac{\omega}{\omega_{B}} e_{n+1} \quad \bmod 2 \pi .
\end{aligned}
$$

Then, letting $w(e, n)$ be the energy distribution, we write the FPK equation corresponding to the map (110)

$$
\begin{aligned}
\frac{\partial w(e, n)}{\partial n} & =\frac{1}{2} \frac{\partial^{2}}{\partial e^{2}}(D(e) w)+\frac{\partial}{\partial e}\left(\alpha e^{3}\right) w, \\
D(e) & =Q^{2} e^{4 / 3} / 2,
\end{aligned}
$$

where $n$ is the iteration number. Now the evolution of observable functions can be described by the FPK equation for the moments of $w(e, n), M(m)=\int e^{m} w(e, n) d e$. In this way we have revealed the relaxation time to a stationary state with the mean value

$$
\langle e\rangle=e_{0}=\left(Q^{2} / 4 \alpha\right)^{3 / 8},
$$

which is equal to $t_{d}=1 / Q^{3 / 4} \alpha^{5 / 8} v_{B}$, and the mean rate of ECRH is determined by

$\mathrm{d} E / \mathrm{d} t=Q^{2} v_{B} m / e^{2 / 3}$.

It is worth noting that the injection mechanism at low energies, which may be intimately related to particle heating, is the important aspect of cosmic-ray physics. Since the rate of ECRH is proportional to $E^{-2 / 3}$, this is indeed the case. This stationary distribution, provided $w, \partial w / \partial e$ vanish at infinity, is given by

$J(e)=\frac{Q^{2}}{4}\left(e^{4 / 3} \frac{\partial w}{\partial e}+\frac{4}{3} e^{1 / 3} f\right)+\alpha e^{3} w=0$, with solution

$w(e)=\frac{\text { const }}{e^{4 / 3}} \exp \left(-\frac{3}{8}\left(\frac{e}{e_{0}}\right)^{8 / 3}\right)$,

where $e_{0}$ is a characteristic energy given by Eq. (112). This equation predicts that the power law will depend on $w(e) \propto$ $e^{-4 / 3}$ at low energies $e<e_{0}$.

Choosing the following values of quantities $B=3 \mu \mathrm{G}, \quad \nu_{B}=8 \mathrm{~Hz}, \quad$ and $\quad \omega_{\mathrm{p}}=2 \pi \cdot 8 \times 10^{2} \mathrm{~s}^{-1}$, we evaluate $e_{0}=10^{8}\left(E_{0}=50 \mathrm{TeV}, \quad t_{\mathrm{d}} \approx 5 \times 10^{3} \mathrm{yr}\right.$, $\left.\mathrm{d} E / \mathrm{d} t \approx 100 / e^{2 / 3} \mathrm{MeVs}^{-1}\right)$, and the parameters $Q=5-10$, $\alpha=5 \times 10^{-21}$. Numerical investigations of Eqs. (110) at these parameters gave the results shown in Fig. 11. This figure indicates the spectrum is bounded both above and below the values of $e_{\mathrm{b}} \approx 4 \times 10^{8}\left(E_{\mathrm{b}} \approx 200 \mathrm{TeV}\right)$ and $E_{\mathrm{c}}(\approx 14 \mathrm{eV})$ defined by Eq. (106). In Fig. 11b, the analytical expression (115) is compared with the numerical results. The numerical points of the spectrum in the main range $E<E_{0}$ all fall full on the curve $w(e)=$ const $\cdot E^{-4 / 3}$ as predicted by (115). Then when one takes into account the geometry of problem (Fig. 10) and assume the distribution along the line of sight is isotropic, we can write the differential flux of electrons as

$\mathrm{d} N / \mathrm{d} E \mathrm{~d} \Omega=(c / 2 \pi) N_{\mathrm{e}}\left(E_{\mathrm{c}}\right)\left(r\left(E_{0}\right) / r\right) w(E)$

with the gyroradius $r$ proportional to $E, w(E)$ given by Eq. (115), and where $N_{\mathrm{e}}\left(E_{\mathrm{c}}\right)$ is the density of resonant electrons at $E=E_{\mathrm{c}}$. If we substitute $w(E)$ in Eq. (116), this expression becomes

$\mathrm{d} N / \mathrm{d} E \mathrm{~d} \Omega=$ const. $E^{-7 / 3} \exp \left[-(3 / 8)\left(E / E_{0}\right)^{8 / 3}\right]$,

with the spectral index $\sim 2.33$ in the range of low energies, $E<E_{0}$.

Since the electrons radiating their peak spectral power at frequency $v$ have energies $e=\sqrt{v / v_{\mathrm{s}}}, v_{\mathrm{s}}=1.26 \times 10^{6} \mathrm{~B}$, we find that the spectral flux density of radiating electrons with the energy spectrum (117) is

$P_{\nu}=$ const. $v^{-2 / 3} \exp \left[-(3 / 8)\left(v / v_{0}\right)^{4 / 3}\right]$,

where $v_{0}=v_{s} e_{0}^{2}\left(\approx 4 \times 10^{16} \mathrm{~Hz}\right)$. Note, the mean radio spectral index is about 0.5 (Reynolds, 2001), or 0.6 (Stevenson and Green, 2002) with a spread of order 0.2.

Inverse Compton scattering of cosmic microwave background (CMB) photons by relativistic electrons is a plausible explanation for $\mathrm{TeV} \gamma$-ray emission from SNRs. We denote by $e_{\mathrm{p}}$ the energy of a primary CMB photon $\left(e_{\mathrm{p}} \simeq 10^{-3} \mathrm{eV}\right)$, and $E_{\gamma}$ as the energy of a $\gamma$-photon. It is well known (Ginzburg, 1989) that electrons with energies $e \ll m / 4 e_{\mathrm{p}}$ can scatter only soft photons $E_{\gamma}=e_{\mathrm{p}} e^{2}$ that contribute to radio and X-ray spectra, while high-energy electrons $e>m / 4 e_{\mathrm{p}}$ will scatter photons with energies $E_{\gamma} \leq E$, whose spectrum 

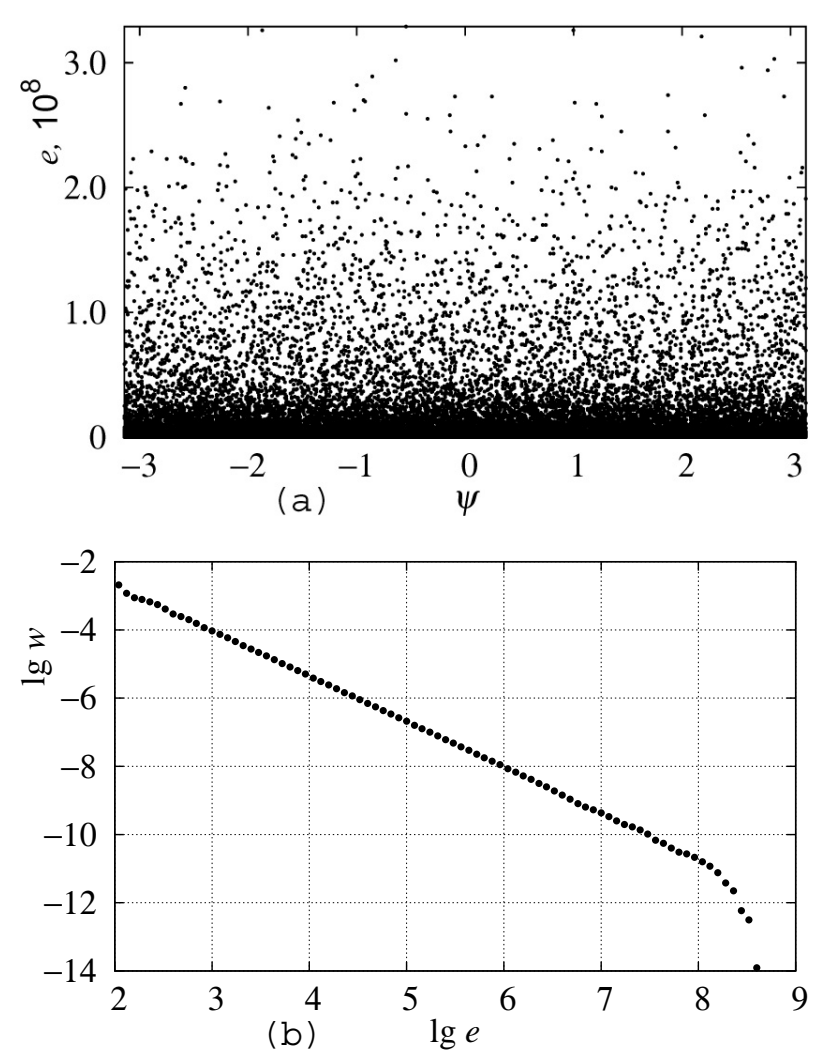

Fig. 11. (a) Phase space $e-\psi$ and (b) the energy distribution $w(e)$ for the map (110) with parameters $Q=9.2, \alpha=10^{-21}$, and $e_{c}=10^{2}$.

is similar to the energy spectrum of electrons. Note that this characteristic energy $\left(m / 4 e_{\mathrm{p}} \approx 10^{8}\right)$ is comparable to the mean energy of electrons. In this case, Compton losses in fact are insensitive to the energy of electrons, and an electron loses its energy with the rate $\mathrm{d} e / \mathrm{d} t=5 \times 10^{-4} \mathrm{~s}^{-1}$ (Ginzburg, 1989). To find the spectrum of $\mathrm{TeV} \gamma$-photons, we first modify this expression as it has been done above, then after substituting in the FPK equation, the following expression results:

$P_{\nu}=$ const. $v^{-2 / 3} \exp \left[-(3 / 2)\left(E_{\gamma} / E_{0}\right)^{2 / 3}\right]$.

The tail of the equation describes $\mathrm{TeV} \gamma$-photon spectrum. This spectrum is quite softer than that given by expression (118); the signature of the spectra observed in SNRs (Reynolds, 2001; Vink, 2004).

Our results allow the inference of an efficiency of shock acceleration of electrons via the electron cyclotron resonance heating and indicate that stochastic surfing acceleration is likely to play an important role in producing relativistic electrons at an SNR shock.

\subsection{Stochastic heating of cosmic-ray electrons by a Langmuir wave packet}

The interaction of relativistic particles with Langmuir waves is thought to be an important mechanism for producing cosmic rays (Kaplan and Tsytovich, 1973; Ginzburg, 1989). According to Ginzburg (1989), the rate of the Galactic beamplasma instability, which generates Langmuir waves with the frequency $\omega_{\mathrm{p}}=3 \times 10^{4} \mathrm{~s}^{-1}$ and minimal wavenumber $k=$ $10^{-6} \mathrm{~cm}^{-1}$ is about $10^{-8} \mathrm{~s}^{-1}$. Thus the time-space scales of such a Langmuir wave packet are likely to be $T=10^{8} \mathrm{~s}$ and $L=3 \times 10^{18} \mathrm{~cm}$, respectively. As long as the group velocity of Langmuir waves is much more less than the speed of light, we concern ourselves with the problem of the nonlinear interaction of relativistic electrons with a spacelike (SL) wave packet provided the Cherenkov resonance condition $k p_{z} / E-\omega=0$ is met. A particle moving in this wave field is governed by the equations

$$
\begin{aligned}
\dot{p}_{z} & =\left(k \varphi_{0} / \sqrt{\Delta k L}\right) \sin \psi L \sum_{n} \delta(z-n L), \\
\dot{\psi} & =k p_{z} / E-\omega_{p} .
\end{aligned}
$$

In the usual way we reduce these equations into the map

$$
\begin{aligned}
E_{n+1} & =E_{n}+\sqrt{(k / \Delta k) k L \varphi_{0}^{2}} \sin \psi_{n}, \\
\psi_{n+1} & =\psi_{n}+k L \\
& -\omega_{p} L\left(E_{n+1} / \sqrt{E_{n+1}^{2}-m^{2}}\right) \quad \bmod 2 \pi,
\end{aligned}
$$

which was introduced by Klimov and Tel'nikhin (1995) and Krotov and Tel'nikhin (1998).

In the nonrelativistic limit, these equations reduce to the well-known map

$$
\begin{aligned}
& E_{n+1}=E_{n}+\sqrt{(k / \Delta k) k L \varphi_{0}^{2}} \sin \psi_{n}, \\
& \psi_{n+1}=\psi_{n}+k L-\text { const. } / \sqrt{E_{n+1}}, \quad E=p^{2} / 2 m,
\end{aligned}
$$

which describes the heating of particles in an SL wave packet of electrostatic waves (Sagdeev et al., 1988). In the contrary limit, $(E / m)^{2} \gg 1$, map (120) in terms of the variables $(\psi, u)$ becomes

$$
\begin{aligned}
g^{n}: \quad & u_{n+1}=u_{n}+Q \sin \psi_{n}, \\
& \psi_{n+1}=\psi_{n}+1 / 2 u_{n+1}^{2} \operatorname{sign} u \bmod 2 \pi, \\
u= & E p_{z} /\left|p_{z}\right| m \sqrt{k L}, \quad Q=\sqrt{(k / \Delta k) U_{0}^{2}}, \\
U_{0}= & \varphi_{0} / m .
\end{aligned}
$$

Numerical investigations of the map at $Q \ll 1$ indicated the appearance of the strange attractor (SA), shown in Fig. 12. The upper bound of the SA is given by

$u_{\mathrm{b}}=Q^{1 / 3}$, 


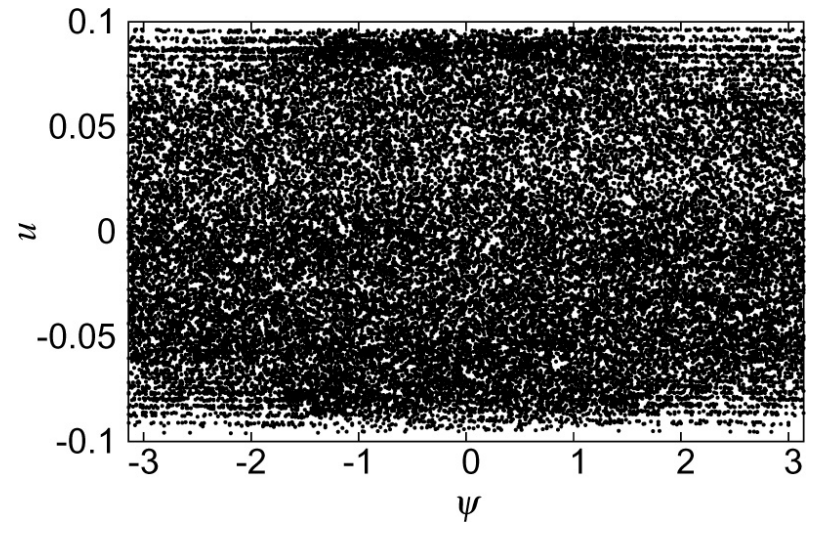

Fig. 12. Strange attractor of the map (121) at $Q=1.1 \times 10^{-3}$.

which is borne out numerically. The energies over which heating can occur are limited to those given by $E_{\mathrm{b}}=$ $m \sqrt{k L} Q^{1 / 3}\left(\sim 100 \mathrm{GeV}\right.$ at $\left.Q=10^{-3}\right)$.

The equations from which the SA arises are usually parameterized by some control parameter whose variation changes the character of the dynamics. When the control parameter $Q$ becomes greater than a critical value $Q_{\mathrm{c}}$, the system exhibits intermittent chaotic behavior shown in Fig. 13. We are already familiar with this kind of dynamics; however, we have never seen such a curious behavior before. It appears that after a finite number of iterations the system demonstrates an intermittent transition to regular unbounded motion. Following the approach of Khazanov et al. (2008b), we describe the motion as the composition $G^{t} \circ g^{n}$ (Arnold, 1989), where $g^{n}$ is the map (121), and $G^{t}$ is the Hamiltonian flow acting on a smooth manifold $M^{2}$. The goal is to find this $G^{t}$. The action of $G^{t}$ is associated with an adiabatic motion, which is realized when the following relations

$$
3 K / 4 \pi u^{2 / 3} \ll 1, \quad Q / u \ll 1, \quad \sin \psi=0
$$

are valid. Now the evolution of the orbit's phase flow is described by the vector field

$\boldsymbol{v}=Q \sin \psi \partial / \partial u+\left(1 / 2 u^{2}\right) \partial / \partial \psi$

on which the family of invariant curves is given by

$h=Q \cos \psi-1 / 2 u$.

This $h$ serves also as a Hamiltonian function of dynamical system (125), the proper values of which are defined by the initial values of $(\psi, u)$ on the SA. Because the behavior of the system is completely determined by these proper values of $h$, we can find the density of states in the space of parameters, $\rho(h)=\tau=\mathrm{d} \Gamma / \mathrm{d} h$, where $\tau=t / T$ is the time of motion along the orbit, and $\Gamma(h)=\oint_{C_{h}} u(h) \mathrm{d} \psi$ is the phase area bounded by the invariant curve $C_{h}$ (Arnold, 1988). Invoking
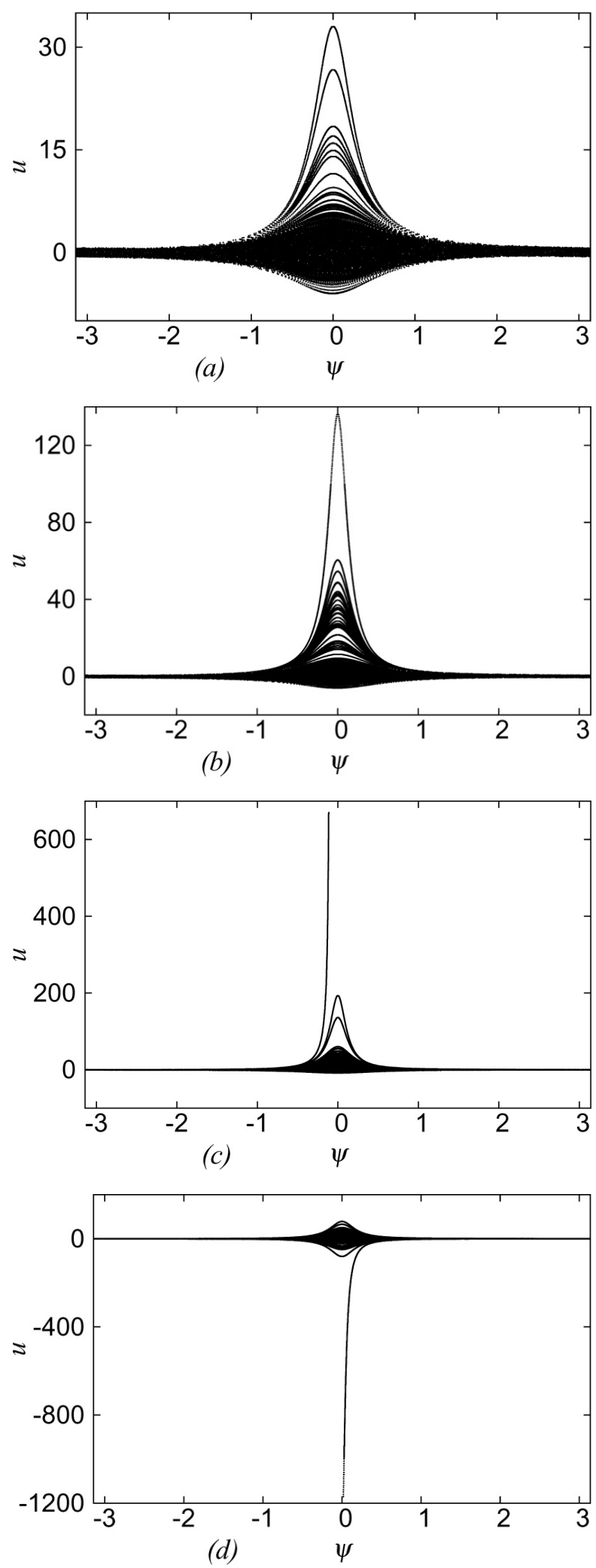

Fig. 13. Phase space $u, \psi$ for the map (121) at $Q=0.367$. Number of iterations increasing from (a)-(c) and (d) as $N=4.2 \times 10^{4} ; 8 \times$ $10^{4} ; 2.2 \times 10^{5}$. 
Eq. (126), we obtain

$\rho(h)=\tau=2 \pi|h| /\left(h^{2}-Q^{2}\right)^{3 / 2}, \quad|h| \geq Q$.

Arising singularity in distribution (127) at $|h|=Q$, we can characterize its $\varepsilon$-vicinity. As the radius of the $\varepsilon$-vicinity tends to 0 , it makes the mean density tends to infinity. As a consequence, the states $|h|=Q$ are the most likely states of the system. In finding an explicit expression for $Q_{\mathrm{c}}$, we use Eq. (126) with $|h|=Q$ to find $\left|u_{\min }\right|=1 / 4 Q$. So far as all these orbits start from the SA, the $u_{\min }$ belongs to the SA. Then by equating $u_{\min }$ to the $u_{\mathrm{b}}$ given by Eq. (123), we obtain $Q_{\mathrm{c}}=1 / 2 \sqrt{2}$, which matches quite well to the numerical values. If we substitute $Q_{\mathrm{c}}$ in (122), assuming $k / \Delta k=100$, and $k=10^{-6} \mathrm{~cm}^{-1}$, these required magnitudes of wave field turn out to be $U_{\mathrm{c}} \simeq 3 \times 10^{-2}$, and $\left(E_{1}\right)_{\mathrm{c}} \sim 10^{-2} \mathrm{mV} \mathrm{cm}^{-1}$.

Now we formulate the problem of dynamics in terms of the variables $(u, \dot{u})$. These variables allow us to obtain more fine information about the geometric structure of phase space. Carrying out the procedure, we derive the following equations:

$W=\frac{1}{2} \dot{u}^{2}+w(u), \quad w(u)=h / 2 u+1 / 8 u^{2}$,

where $W$ is an invariant of motion. To our surprise, we run up against the old Kepler problem here. This can be seen directly from (128) by defining the effective potential $w(u)$. It is worth noting that this problem, unlike the Kepler one, is invariant under the transformation $(-h, u) \rightarrow(h,-u)$. Until the proper values of $W$ are less than 0 , the system exhibits bounded motion; in another case, which is realized for example, at $h=-Q$ and $u_{\min }=1 / 4 Q$, the motion becomes unbounded. Strange as it may appear, the singularity in distribution (127) leads to a topological modification of phase space. At first, changes in topology emerge provided the conditions $Q>Q_{\text {c }}$ but $|h|>Q$ hold. In this case, the topological space is a direct sum of the strange attractor and torus. There is another change in topology at $|h|=Q$. As it appears from Eqs. (125)-(126), the trajectory approaches infinity at $\psi=0$, or $\pm \pi$, and the vector field vanishes. It is known, on the other side, any vector field on a torus vanishes nowhere (Arnold, 1989); consequently this kind of dynamics is associated with a metamorphism of phase space.

Now we consider the dynamics of the system in the offresonant case, i.e., when the phase velocities of the waves in the packet differ from the speed of light. Setting $\left(\omega_{p} T-\right.$ $k L) \bmod 2 \pi=\Omega, \Omega>0$, to be the phase shift in the phase advance equation (121), we numerically investigate the behavior of the system. Shown in Fig. 14 is the phase space evolution of the system. We cannot resist the fascination of the strange behavior. Trying to understand it, we write the phase flow associated with the Hamiltonian,

$h=Q \cos \psi-1 / 2 u-\Omega$,
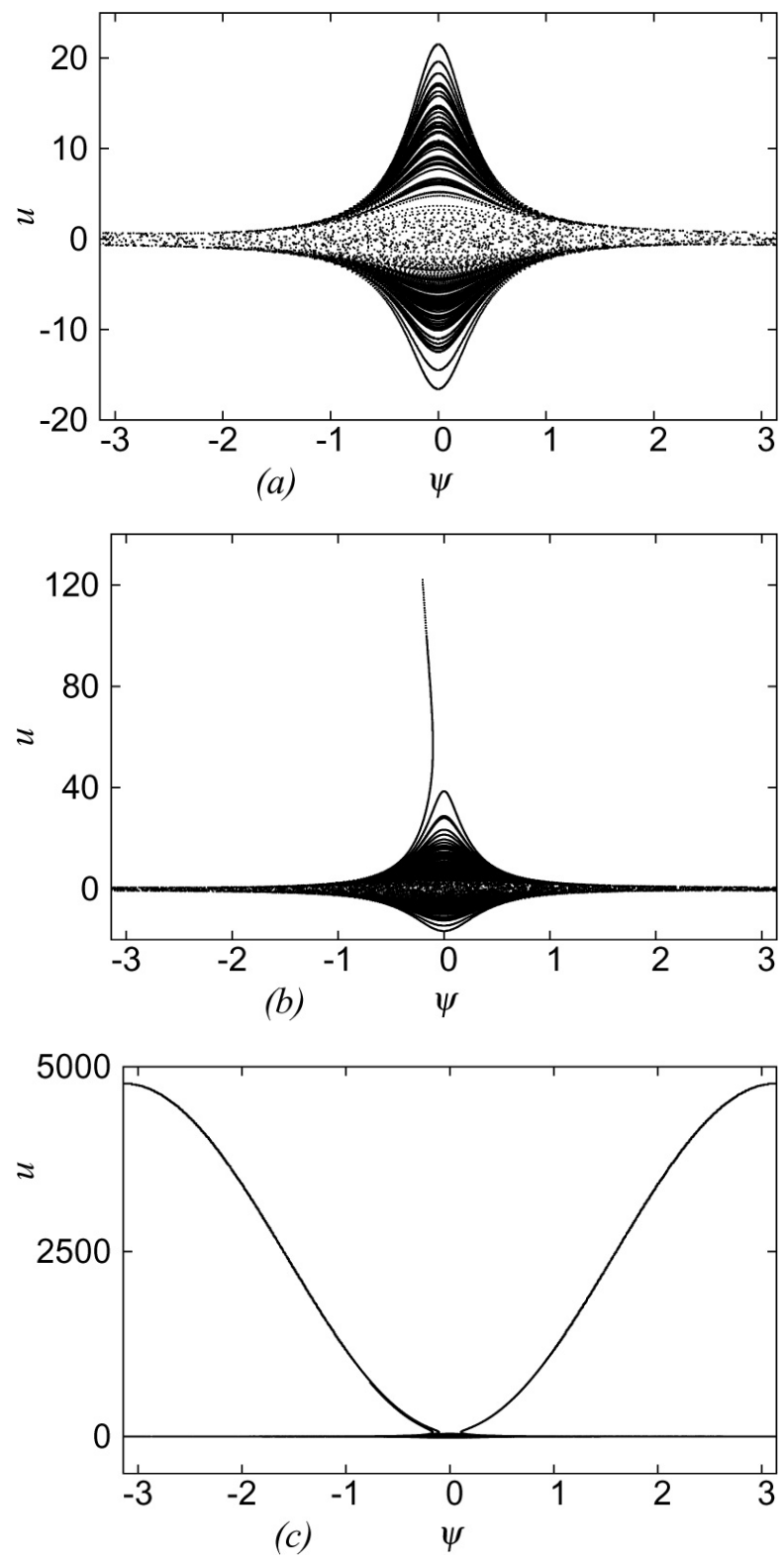

Fig. 14. Dynamics of the system (121) in the off-resonant case. Here $Q=0.367$ and $w=\pi / 2 \times 10^{-4}$. (a) $2 \cdot 10^{4}$, (b) $3.5 \times 10^{4}$, and (c) $8 \times 10^{4}$ iterations of a single initial condition.

in the form

$\dot{u}=Q \sin \psi, \dot{\psi}=-\Omega+1 / 2 u^{2}$.

The proper values of $h$ in turn determine a family of invariant curves on the vector field

$\boldsymbol{v}=Q \sin \psi \partial / \partial u+\left(1 / 2 u^{2}-\Omega\right) \partial / \partial \psi$.

It is easily seen that the function $h$ changes its sign, vector field (131) is invariant under the transformation

$u \rightarrow-u, \psi^{\prime} \rightarrow \psi+\pi$, 
and the fixed points of the vector field are given by

$\psi_{0}=0,\left|u_{0}\right|=1 / \sqrt{2 \Omega}$.

One considers these dynamics in a configuration space proceeding to the variable $(u, \dot{u})$. The procedure for deriving the invariant of motion

$W=\frac{1}{2} \dot{u}^{2}+w(u), w(u)=h / 2 u+1 / 8 u^{2}+h \Omega u+\Omega^{2} u^{2} / 2$,

is the same at that used for obtaining (128). This potential is symmetric with respect to $(-h, u) \rightarrow(h,-u)$, and the turning points are situated next to $\left|u_{\min }\right|=1 / 4 Q,\left|u_{\max }\right|=$ $2 Q / \Omega$. These $u_{\max }$ determine a monochromatic energy state $E_{\text {max }}=2 m \sqrt{k L} Q / \Omega$ with different directions of $p_{z}$ momentum. The potential $w(u)$ has a local maximum at the fixed points, and the motion near these points obeys the equation $\ddot{\psi}+\left(Q / u_{0}^{3}\right) \sin \psi=0$, which shows just the same invariance (132), and the tangent vector at a point of fixed points changes its direction. Thus there are two allowable local coordinate systems $\left(\psi^{\prime}, u^{\prime}\right)$ and $(\psi, u)$, whose determinant of the transformation matrix from one to another, $\left|\begin{array}{cc}\partial \psi^{\prime} / \partial \psi & 0 \\ 0 & \partial u^{\prime} / \partial u\end{array}\right|$, is equal to -1 . There is very good reason to believe that this manifold is a direct sum of the strange attractor and the Mobius strip. In the resonant case, the width of this strip approach infinity. Therefore, the off-resonant effect eliminates a possibility of unbounded motion. To describe the effect of synchrotron emission and inverse Compton scattering on the dynamics, we again apply the method developed above (Sect. 4.1, formula (109)). In this way we derive the following equations

$u_{n+1}=u_{n}\left(1-\alpha\left|u_{n}\right|\right)+Q \sin \psi_{n}$,

$\psi_{n+1}=1 / 2 u_{n+1}^{2} \operatorname{sign} u(\bmod 2 \pi)$,

where $\alpha=\beta_{\mathrm{s}} T \sqrt{k L}\left(\sim 8 \times 10^{-6}\right)$, and all another variables and the parameter $Q$ remain the same. When the wave perturbation is small, $Q \sim 10^{-3}$, the system exhibits chaotic behavior on a strange attractor. For the dissipative system the phase space volume contracts as the motion proceeds. In virtue of it, the topology of the SA is quite remarkable, showing the property of fractional dimensionality, $d \simeq$ $2-2 \alpha Q^{1 / 3} / 3 h_{k}$, where $h_{k}$ is the Kolmogorov entropy of the original Hamiltonian system. For the parameter range of interest, numerical investigations of the system motion are shown in Fig. 15. The figure displays the emphatic intermittent behavior. It also proves possible to represent the phase flow as

$$
\begin{aligned}
& \dot{u}=-\alpha u^{2}+Q \sin \psi, \\
& \dot{\psi}=1 / 2 u^{2},
\end{aligned}
$$

the fixed points of which are given by the equation

$\alpha u_{0}^{2}=Q \sin \psi_{0}$.

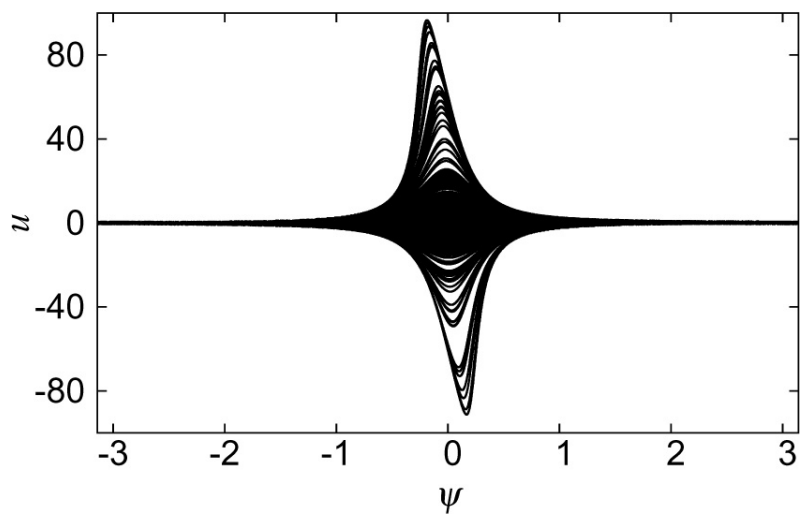

Fig. 15. Phase space for dissipative map (135) with $Q=0.4$ and $\alpha=8 \times 10^{-6}$.

Equation (136) defines the phase shift away from the resonance, and in implicit form determines the range of accessible proper values of $u, u<\sqrt{Q / \alpha}(\sim 200)$ in virtue of Eq. (122); the upper value of energy should be about $100 \mathrm{TeV}$. The estimates seem to verify this inference.

We propound the model of the stochastic dynamics of relativistic electrons driven by the Langmuir wave packet. Whether or not a model like this can be applied to the cosmicray electron acceleration, seemingly, will be a problem for future research, when such a kind of model will need to be compared with data at this level of precision to give confidence in the results (Reynolds, 2001).

\section{Conclusions}

We study the motion of relativistic electrons interacting resonantly with space-plasma waves. The nonlinear resonant interaction is shown to originate electron chaotic motion related to the profound change in topology of phase space, and the appearance of the strange attractor. In this case, every particle can explore the entire area of phase space energetically accessible to it; as a result, the upper bound of the strange attractor can be put on a one-to-one correspondence with the upper boundary of an energy spectrum whose value depends parametrically on the spectral power of the wave. The chaotic motion on the strange attractor is ergodic with mixing, and as a consequence, the evolution of the distribution function and all means obeys a FPK equation. In nonlinear theory, the rate of diffusion as a function of wave power energy hinges on the type of interaction. These dependencies have important consequences in determining the range of values of wave power for which the FPK formalism is valid. The results are applied to the problem of acceleration of cosmic-ray electrons and the Earth's radiation belt electrons. 
Acknowledgements. Funding in support of this study was provided by NASA grant UPN 370-16-10, the NASA HQ POLAR project, and the NASA LWS program.

Edited by: G. Morales

Reviewed by: two anonymous referees

\section{References}

Abdo, A. A., Ackermann, M., Ajello, M., Asano, K., Atwood, W. B., Axelsson, M., Baldini, L., Ballet, J., Barbiellini, G., Baring, M. G., Bastieri, D., Bechtol, K., Bellazzini, R., Berenji, B., Bhat, P. N., Bissaldi, E., Blandford, R. D., Bloom, E. D., Bonamente, E., Borgland, A. W., Bouvier, A., Bregeon, J., Brez, A., Briggs, M. S., Brigida, M., Bruel, P., Burgess, J. M., Burrows, D. N., Buson, S., Caliandro, G. A., Cameron, R. A., Caraveo, P. A., J., Casandjian, M., Cecchi, C., Çelik, Ö., Chekhtman, A., Cheung, C. C., Chiang, J., Ciprini, S., Claus, R., Cohen-Tanugi, J., Cominsky, L. R., Connaughton, V., Conrad, J., Cutini, S., d'Elia, V., Dermer, C. D., de Angelis, A., de Palma, F., Digel, S. W., Dingus, B. L., do Couto e Silva, E., Drell, P. S., Dubois, R., Dumora, D., Farnier, C., Favuzzi, C., Fegan, S. J., Finke, J., Fishman, G., Focke, W. B., Fortin, P., Frailis, M., Fukazawa, Y., Funk, S., Fusco, P., Gargano, F., Gehrels, N., Germani, S., Giavitto, G., Giebels, B., Giglietto, N., Giordano, F., Glanzman, T., Godfrey, G., Goldstein, A., Granot, J., Greiner, J., Grenier, I. A., Grove, J. E., Guillemot, L., Guiriec, S., Hanabata, Y., Harding, A. K., Hayashida, M., Hays, E., Horan, D., Hughes, R. E., Jackson, M. S., Jóhannesson, G., Johnson, A. S., Johnson, R. P., Johnson, W. N., Kamae, T., Katagiri, H., Kataoka, J., Kawa, N., Kerr, M., Kippen, R. M., Knödlseder, J., Kocevski, D., Komi, N., Kouveliotou, C., Kuss, M., Lande, J., Latronico, L., Lemoine-Goumard, M., Longo, F., Loparco, F., Lott, B., Lovellette, M. N., Lubrano, P., Madejski, G. M., Makeev, A., Mazziotta, M. N., McBreen, S., McEnery, J. E., McGlynn, S., Meegan, C., Mészáros, P., Meurer, C., Michelson, P. F., Mitthumsiri, W., Mizuno, T., Moiseev, A. A., Monte, C., Monzani, M. E., Moretti, E., Morselli, A., Moskalenko, I. V., Murgia, S., Nakamori, T., Nolan, P. L., Norris, J. P., Nuss, E., Ohno, M., Ohsugi, T., Omodei, N., Orlando, E., Ormes, J. F., Paciesas, W. S., Paneque, D., Panetta, J. H., Pelassa, V., Pepe, M., Pesce-Rollins, M., Petrosian, V., Piron, F., Porter, T. A., Preece, R., Rainò, S., Rando, R., Rau, A., Razzano, M., Razzaque, S., Reimer, A., Reimer, O., Reposeur, T., Ritz, S., Rochester, L. S., Rodriguez, A. Y., Roming, P. W. A., Roth, M., Ryde, F., Sadrozinski, H. F.-W., Sanchez, D., Sander, A., Saz Parkinson, P. M., Scargle, J. D., T. L., Schalk, C. Sgrò, E. J., Siskind, P. D., Smith, P., Spinelli, Stamatikos, M., Stecker, F. W., Stratta, G., Strickman, M. S., Suson, D. J., Swenson, C. A., Tajima, H., Takahashi, H., Tanaka, T., Thayer, J. B., Thayer, J. G., Thompson, D. J., Tibaldo, L., Torres, D. F., Tosti, G., Tramacere, A., Uchiyama, Y., Uehara, T., Usher, T. L., van der Horst, A. J., Vasileiou, V., Vilchez, N., Vitale, V., von Kienlin, A., Waite, A. P., Wang, P., Wilson-Hodge, C., Winer, B. L., Wood, K. S., Yamazaki, R., Ylinen, T., and Ziegler, M.: Fermi Observations of GRB 090902B: A Distinct Spectral Component in the Prompt and Delayed Emission, Astrophys. J. Lett., 706, L138 doi:10.1088/0004-637X/706/1/L138, 2009.
Albert, J. M.: Nonlinear interaction of outer zone electrons with VLF waves, Geopys. Res. Lett., 29, 116-1-116-3, doi:10.1029/2001GL013941, 2002.

Arnold, V.: Geometrical Methods in the Theory of Ordinary Differential Equations, Springer-Verlag, New York, 1988.

Arnold, V.: Mathematical Methods of Classical Mechanics, Springer-Verlag, New York, 1989.

Arnold, V. and Avez, A.: Ergodic Problems of Classical Mechanics, Benjamin, New York, 1968.

Artsimovich, L. and Sagdeev, R.: Plasma Physics for Physicists, Atom Press, Moscow, 1979 (in Russian).

Bell, A. R.: The acceleration of cosmic rays in shock fronts, Mon. Not. Roy. Astron. Soc., 182, 147-156, 1978.

Bernstein, I., Greene, J. M., and Kruskal, M. D.: Exact Nonlinear Plasma Oscillations, Phys. Rev., 108, 546-550, doi:10.1103/PhysRev.108.546, 1957.

Blake, J. B., Baker, D. N., Turner, N., Ogilvie, K. W., and Lepping, R. P.: Correlation of changes in the outer-zone relativistic-electron population with upstream solar wind and magnetic field measurements, Geophys. Res. Lett., 24, 927-929, doi:10.1029/97GL00859, 1997.

Blandford, R. and Ostriker, J.: Particle acceleration by astrophysical shocks, Astrophys. J., 221, L29-L32, 1978.

Cargill, P. J. and Papadopoulos, K.: A mechanism for strong shock electron heating in supernova remnants, Astrophys. J., 329, L29L32, doi:10.1086/185170, 1988.

Cattell, C., Wygant, J. R., Goetz, K., Kersten, K., Kellogg, P. J., von Rosenvinge, T., Bale, S. D., Roth, I., Temerin, M., Hudson, M. K., Mewaldt, R. A., Wiedenbeck, M., Maksimovic, M., Ergun, R., Acuna, M., and Russell, C. T.: Discovery of very large amplitude whistler-mode waves in Earth's radiation belts, Geophys. Res. Lett., 35, L01105, doi:10.1029/2007GL032009, 2008.

Chernikov, A. A., Tel, T., Vattay, G., and Zaslavsky, G. M.: Chaos in the relativisti generalization of the standard map, Phys. Rev. A, 40, 4072-4076, doi:10.1103/PhysRevA.40.4072, 1989.

Chirikov, B.: A universal instability of many-dimensional oscillator systems, Phys. Rep., 52, 263-379, 1979.

Cully, C. M., Bonnell, J. W., and Ergun, R. E.: THEMIS observations of long-lived regions of large-amplitude whistler waves in the inner magnetosphere, Geophys. Res. Lett., 35, L17S16, doi:10.1029/2008GL033643, 2008.

Dieckmann, M., Eliasson, B., and Shukla, P.: Self-consistent Studies of Electron Acceleration to Ultrarelativistic Energies by Upper Hybrid Waves, Astrophys. J., 617, 1361-1370, doi:10.1086/425568, 2004.

Faith, J., Kuo, S., and Huang, J.: Electron precipitation caused by chaotic motion in the magnetosphere due to largeamplitude whistler waves, J. Geophys. Res., 102, 2233-2241, doi:10.1029/96JA01968, 1997.

Ginzburg, V.: Applications of Electrodynamics in Theoretical Physics and Astrophysics, Gordon and Breach, New York, 1989.

Green, J. C., Onsager, T. G., O'Brien, T. P., and Baker, D. N.: Testing loss mechanisms capable of rapidly depleting relativistic electron flux in the Earth's outer radiation belt, J. Geophys. Res., 109, A12211, doi:10.1029/2004JA010579, 2004.

Hastings, D. and Garrett, H.: Spacecraft-Environment Interactions, Cambridge University Press, New York, 1996.

Horton, W. and Ichikawa, Y. H.: Chaos and Structures in Nonlinear Plasmas, World Scientific, Singapore, 1996. 
Kaplan, S. A. and Tsytovich, V. N.: Plasma Astrophysics, Pergamon Press, Oxford, 1973.

Karpman, V.: Nonlinear Waves in Dispersive Media, Pergamon, New York, 1975.

Kaup, D. J. and Newell, A. C.: Solitons as Particles, Oscillators, and in Slowly Changing Media: A Singular Perturbation Theory, P. R. Soc. A, 361, 413-446, doi:10.1098/rspa.1978.0110, 1978.

Kellogg, P. J., Cattell, C. A., Goetz, K., Monson, S. J., and Wilson III, L. B.: Large amplitude whistlers in the magnetosphere observed with Wind-Waves, J. Geophys. Res., 116, A09224, doi:10.1029/2010JA015919, 2011.

Kersten, K., Cattell, C. A., Breneman, A., Goetz, K., Kellogg, P. J., Wygant, J. R., Wilson III, L. B., Blake, J. B., Looper, M. D., and Roth, I.: Observation of relativistic electron microbursts in conjunction with intense radiation belt whistler-mode waves, Geophys. Res. Lett., 38, L08107, doi:10.1029/2011GL046810, 2011.

Khazanov, G., Tel'nikhin, A., and Kronberg, T.: Dynamic theory of relativistic electrons stochastic heating by whistler mode waves with application to the Earth magnetosphere, J. Geophys. Res., 113, A03207, doi:10.1029/2007JA012488, 2008a.

Khazanov, G., Tel'nikhin, A., and Kronberg, T.: Nonlinear electron motion in a coherent whistler wave packet, Phys. Plasmas, 15, 073506, doi:10.1063/1.2959121, 2008b.

Khazanov, G., Tel'nikhin, A., and Kronberg, T.: Bursty precipitation driven by chorus waves, in: Radiation Effects and Defects in Solids: Incorporating Plasma Science and Plasma Technology, Special Issue: Plasma: Fusion and Environments, 166, 795-805, doi:10.1080/10420150.2011.610319, 2011.

Khazanov, G. V.: Kinetic Theory of the Inner Magnetosheric Plasma, Astrophysics and Space Science Library 372, Springer Science, New York, 2011.

Khazanov, G. V., Tel'nikhin, A., and Kronberg, T.: Chaotic motion of relativistic electrons driven by whistler waves, Plasma Phys. Contr. F., 49, 447-466, doi:10.1088/0741-3335/49/4/008, 2007.

Khazanov, G. V., Tel'nikhin, A. A., and Krotov, A.: Stochastic ion heating by the lower-hybrid waves, in: Radiation Effects and Defects in Solids: Incorporating Plasma Science and Plasma Technology, Special Issue: Plasma Science and Plasma Technology, 165, 165-176, doi:10.1080/10420150903516684, 2010

Klimov, O. and Tel'nikhin, A.: Stochastic dynamics of a relativistic particle in the field of a wave packet, Plasma Phys. Rep., 21, 335-338, 1995.

Krotov, A. and Tel'nikhin, A.: Stochastic heating of relativistic particles in the field of a wide wave packet, Plasma Phys. Rep., 24, 767-771, 1998

Landau, L. and Lifshitz, E.: The Classical Theory of Fields, vol. 2, Butterworth-Heinemann, Waltham, 1980.

Lichtenberg, A. and Lieberman, M.: Regular and Chaotic Dynamics, Springer, New York, 1992.

Liemohn, M. W.: Introduction to special section on "Results of the National Science Foundation Geospace Environment Modeling Inner Magnetosphere/Storms Assessment Challenge", J. Geophys. Res., 111, A11S01, doi:10.1029/2006JA011970, 2006.

Liu, S., Fan, Z., Fryer, C., Wang, J., and Li, H.: Stochastic Electron Acceleration in Shell-Type Supernova Remnants, Astrophys. J. Let.., 683, L163-L166, doi:10.1086/591793

Lutomirski, R. and Sudan, R.: Exact Nonlinear Electromagnetic Whistler Modes, Phys. Rev., 147, 156-165, doi:10.1103/PhysRev.147.156, 1966.
Lyons, L. and Williams, D.: Quantitative aspects of magnetospheric physics, Reidel, Boston, 1984.

Muranushi, T. and Inutsuka, S.: Direct Simulations of Particle Acceleration in a Fluctuating Electromagnetic Field Across a Shock, Astrophys. J., 691, L24-L26, doi:10.1088/0004637X/691/1/L24, 2009.

Nagornykh, E. and Tel'nikhin, A.: Stochastic Motion of Relativistic Particles in the Field of a Wide Wave Packet, in: 11th International Congress on Plasma Physics: ICPP2002, 15-19 July 2002 , Sydney, Australia, edited by: Falkoner, I. S., Dewar, R. L., and Khachan, J., American Institute of Physics (AIP), AIP Conference Proceedings, 669, 824-827, doi:10.1063/1.1594057, 2003.

Newell, A.: Solitons in Mathematics and Physics, SIAM, Philadelphia, 1985.

Nomura, Y., Ichikawa, Y. H., and Horton, W.: Nonlinear dynamics of the relativistic standard map, Phys. Rev. A, 45, 1103-1115, doi:10.1103/PhysRevA.45.1103, 1992.

O'Brien, T. P., Looper, M. D., and Blake, J. B.: Quantification of relativistic electron microburst losses during the GEM storms, Geophys. Res. Lett., 31, L04802, doi:10.1029/2003GL018621, 2004.

Reynolds, S.: Synchrotron Radiation from Galactic Sources: What We Can Learn About Particle Acceleration, Space Sci. Rev., 99, 177-186, doi:doi:10.1023/A:1013892931294, 2001.

Richtmyer, R.: Principles of Advanced Mathematical Physics, vol. 1, Springer-Verlag, New York, 1978.

Roth, I., Temerin, M., and Hudson, M. K.: Resonant enhancement of relativistic electron fluxes during geomagnetically active periods, Ann. Geophys., 17, 631-638, doi:10.1007/s00585-9990631-2, 1999.

Sagdeev, R., Usikov, D., and Zaslavsky, G.: Introduction to Nonlinear Physics, Harwood, New York, 1988.

Santolik, O., Gurnett, D. A., Pickett, J. S., Parrot, M., and Cornilleau-Wehrlin, N.: Spatio-temporal structure of storm-time chorus, J. Geophys. Res., 108, 1278, doi:10.1029/2002JA009791, 2003.

Schmidt, G.: Physics of High Temperature Plasmas, Academic Press, New York, 1979.

Schutz, B.: Geometrical methods of Mathematical Physics, Cambridge University Press, Cambridge, London, 1982.

Selesnick, R. S.: Source and loss rates of radiation belt relativistic electrons during magnetic storms, J. Geophys. Res., 111, A04210, doi:10.1029/2005JA011473, 2006.

Selesnick, R. S., Blake, J. B., Kolasinski, W. A., and Fritz, T. A.: A quiescent state of 3 to $8 \mathrm{MeV}$ radiation belt electrons, Geophys. Res. Lett., 24, 1343-1346, doi:10.1029/97GL51407, 1997.

Stevenson, F. and Green, D.: Historical Supernovae and Their Remnants, Clarendon Press, Oxford, 2002.

Stix, T.: Waves in Plasmas, AIP Press, New York, 1992.

Summers, D., Ni, B., and Meredith, N. P.: Timescales for radiation belt electron acceleration and loss due to resonant wave-particle interactions: 2. Evaluation for VLF chorus, ELF hiss, and electromagnetic ion cyclotron waves, J. Geophys. Res., 112, A04207, doi:10.1029/2006JA011993, 2007.

Teller, E.: Relativistic hydrodynamics in Supernovae, in: Physics of High Energy Density, edited by: Caldiroba, P. and Knoepfel, H., chap. 15, Academic Press, New York, 1971. 
Treumann, R. A. and Terasawa, T.: Electron Acceleration in the Heliosphere, Space Sci. Rev., 99, 135-150, doi:10.1023/A:1013836813547, 2001.

Tsurutani, B. T., Falkowski, B. J., Verkhoglyadova, O. P., Pickett, J. S., Santolík, O., and Lakhina, G. S.: Quasi-coherent chorus properties: 1. Implications for wave-particle interactions, J. Geophys. Res., 116, A09210, doi:10.1029/2010JA016237, 2011.

Vink, J.: Shocks and particle acceleration in supernova remnants: observational features, Adv. Space Res., 33, 356-365, doi:10.1016/j.asr.2003.05.012, 2004.

Whitham, G. B.: Linear and Nonlinear Waves, Wiley, New York, 1974.

Wilson, L. B., Cattell, C. A., Kellogg, P. J., Wygant, J. R., Goetz, K., Breneman, A., and Kersten, K.: The properties of large amplitude whistler mode waves in the magnetosphere: Propagation and relationship with geomagnetic activity, Geophys. Res. Lett., 38, L17107, doi:10.1029/2011GL048671, 2011.
Wykes, W. J., Chapman, S., and Rowlands, G.: Enhanced phase space diffusion due to chaos in relativistic electronwhistler mode wave particle interactions with applications to Jupiter, Planet. Space Sci., 49, 395-404, doi:10.1016/S00320633(00)00160-4, 2001.

Zaslavsky, G., Natenson, M., Petrovichev, B., Sagdeev, R., and Chernikov, A.: Stochastic acceleration of relativistic particles in magnetic field, Eks. Teor. Fiz. (J. Exp. Theor. Phys.), 93, 1987.

Zheng, Q., Zheng, Y., Fok, M.-C., and Lui, A. T. Y.: Electron energy diffusion and advection due to non-linear electronchorus wave interactions, J. Atmos. Sol.-Terr. Phy., 80, 152-160, doi:10.1016/j.jastp.2012.01.011, 2012.

Zheng, Q., Fok, M.-C., Zheng, Y., and Lui, A. T. Y.: Nonlinear whistler mode wave effects on magnetospheric energetic electrons, J. Atmos. Sol.-Terr. Phy., 102, 8-16, doi:10.1016/j.jastp.2013.03.018, 2013. 